\title{
Normalization Through Invariants in $n$-Dimensional Kepler Problems
}

\author{
Kenneth R. Meyer ${ }^{1 *}$, Jesús F. Palacián ${ }^{* *}$, and Patricia Yanguas ${ }^{2 * *}$ \\ 1 Department of Mathematical Sciences \\ University of Cincinnati \\ Cincinnati, Ohio 45221-0025, USA \\ ${ }^{2}$ Departamento de Estadística, Informática y Matemáticas and Institute for Advanced Materials \\ Universidad Pública de Navarra \\ 31006 Pamplona, Spain
}

Received January 9, 2018; accepted Month XX, 20XX

\begin{abstract}
We present a procedure for the normalization of perturbed Keplerian problems in $n$ dimensions based on Moser regularization of the Kepler problem and the invariants associated to the reduction process. The approach allows us, not only to circumvent the problems introduced by certain classical variables used in the normalization of this kind of problems, but also to do both the normalization and reduction in one step. The technique is introduced for any dimensions and is illustrated for $n=2,3$ by relating Moser coordinates with Delaunay-like variables. The theory is applied to the spatial circular restricted three-body problem for the study of the existence of periodic and quasi-periodic solutions of rectilinear type.
\end{abstract}

MSC2010 numbers: 34C20, 34C29, 34C40, 37J40, 70F10, 70K65

DOI: $10.0000 / \mathrm{S} 1560354700000012$

Keywords: Kepler Hamiltonian in $n$ dimensions, perturbed Keplerian problems, Moser regularization, Delaunay and Delaunay-like coordinates, Keplerian invariants, regular reduction, periodic and quasi-periodic motions, KAM theory for properly degenerate Hamiltonians

\section{To Anatoly P. Markeev on his seventy-fifth birthday}

\section{INTRODUCTION}

We propose a new approach to the analysis of the classic perturbed Kepler problem. We look at the regularized problem in $n$ dimensions with the goal of finding periodic and quasi-periodic solutions as well as related bifurcations by methods which avoid some of the traditional pitfalls. This approach is illustrated with the analysis of several new and old problems.

We give an alternative way of carrying out the normalization of three-dimensional perturbed Keplerian systems by first applying the Moser regularization mapping and then averaging the regularized perturbation with respect to the eccentric anomaly. The approach is easily adapted to the case of $n$-dimensional perturbed Kepler problems by generalizing the invariants of the spatial Kepler problem to any dimension $n$. Our procedure is based on the fact that the regularized perturbed Kepler problem can be written as a Hamiltonian system in a collection of variables given by a pair of action-angle coordinates together with some global coordinates (invariants) that parameterize the reduced space of the $n$-dimensional Kepler problem. This space is the set $G_{2, n+1}$, the Grassmannian manifold of oriented 2-planes in $\mathbb{R}^{n+1}$, see [35]. If $n=2$, then the reduced space becomes $\mathcal{S}^{2}$, whereas it is $\mathcal{S}^{2} \times \mathcal{S}^{2}$ when $n=3$. The normalization can be interpreted as an average with respect to the angle coordinate. One of the advantages of our approach is that after

\footnotetext{
${ }^{*}$ E-mail: ken.meyer@uc.edu

${ }^{* *}$ E-mail: palacian@unavarra.es

*** E-mail: yanguas@unavarra.es
} 
normalization the resulting Hamiltonian is directly expressed in terms of the invariants that define the reduced space $G_{2, n+1}$.

Our procedure is closely related to the constrained normal form result of van der Meer and Cushman [50] (see also [10,12]), where the authors design a method to deal with the normalization of a perturbed Keplerian Hamiltonian in $n$ dimensions in terms of the coordinates introduced after applying Moser regularization [36]. Indeed, the use of these coordinates allows one to treat all type of bounded motions provided the perturbation is a smooth function after regularization. We show the relation between the approach of [50] and ours by giving explicitly the mappings between Moser coordinates and the Keplerian invariants associated to the Kepler reduction. When $n=3$ Pauli [44] in his quantization of the hydrogen atom provided the quantum differential operators that correspond to the angular momentum and the Laplace-Runge-Lenz vectors, and pointed out that they have an so(4) Lie algebra structure. More recently, Cordani [7] has designed a method to normalize perturbed Kepler problems in global coordinates which are somehow related to Moser coordinates and the invariants of $\mathcal{S}^{2} \times \mathcal{S}^{2}$.

Due to the explicit relations we provide between the rectangular coordinates and the Keplerian invariants, compared to other approaches to the subject, we get a deeper insight in the issue of normalization of perturbed Kepler problems for any dimension $n$. Specifically, our procedure allows one to analyze a certain problem from its corresponding reduced system constructed in several steps, namely: (i) regularizing the problem; (ii) normalizing it up to a certain order arriving at the reduced Hamiltonian written in terms of the Keplerian invariants; (iii) building the reduced system (i.e., the equations of motion) and; (iv) analyzing the system in the reduced space taking into account the constraints defining it.

A change of time in the regularization process is indispensable if one wants to analyze the possibility of solutions of collision nature. This occurs in many examples, such as the restricted three-body problem in the space [33, 51]. For other three-body examples see for instance $[41,42,46]$ where the Hamiltonian, after applying the Jacobi transformation to eliminate the translational symmetry, can be written as the sum of two Keplerian Hamiltonians plus a small perturbation dealing with the coupling terms of the two systems.

We have noticed that if we apply Ligon-Schaaf regularization [28], that does not require scaling the time, a given perturbation cannot be expressed in an analytic way with respect to all of LigonSchaaf's coordinates when we are at the collision manifold, even when the Kepler Hamiltonian and the perturbation both make sense for collisions. This problem is overcome if one applies Kustaanheimo-Stiefel (for dimension 3), Levi-Civita (for dimension 3) or Moser (for dimension $n$ ) formalisms. In any of these settings the perturbation is analytic with respect to the symplectic coordinates that define these mappings, and the same happens if we proceed with the invariants of the Kepler problem and the pair of action-angle coordinates that we introduce. The main reason is that we are avoiding the mean anomaly as part of these coordinates since it is essentially replaced by the eccentric anomaly and the perturbation behaves smoothly with respect to it. Thus, one can apply averaging techniques, normal forms and reduction using any of these procedures and study perturbations of the Kepler problem properly.

For the spatial case of the circular restricted three-body problem in the lunar regime we have analyzed the solutions (periodic and quasi-periodic) of rectilinear bounded nature. This has been achieved after checking a transversality condition that is required in order to get true solutions and this issue has been applied in different types of three-body problems [6, 16, 43, 52].

The paper is structured in six sections plus a list of references. Regularization of the $n$ dimensional Kepler problem is recalled in Section 2. In Section 3 the coordinates used by Moser are related to a set of action-angle coordinates deduced from Delaunay elements (the so called Delaunay-like coordinates), whereas Section 4 is devoted to present the normalization procedure in both Delaunay-like coordinates and the invariants associated to the reduction of the Kepler problem. In Section 5 we generalize the normalization procedure to deal with $n$-dimensional cases, stating and proving our two main results (Theorems 1 and 2). Section 6 is dedicated to treat the case of periodic solutions and KAM 3-tori in the spatial restricted three-body problem. It is remarkable that even when we perform a regularization we do not need an isoenergetic version of a KAM theorem since our method allows us to carry out the calculations for all (negative) energy levels at once. 
The relationship between Moser coordinates for $n=3$ and Delaunay-like variables presented in Section 3.1 is new and allows one the normalization of perturbed Kepler problems by means of action-angle variables. The explicit transformation relating rectangular coordinates to the invariants of the reduced space $\mathcal{S}^{2} \times \mathcal{S}^{2}$ presented in Section 4 is also a novelty. This is generalized to dimension $n$ in Section 5. Finally, the procedure of Section 6 to check the transversality of the manifolds in the spatial restricted three-body problem appears here for the first time.

\section{REGULARIZATION OF THE KEPLER PROBLEM}

The linear and inverse square law, the Kepler problem, are unique among central force problems in that they have hidden symmetries beyond the obvious rotational symmetry and extra integrals in addition to angular momentum.

First Kepler observed that the perihelion of Mars was fixed in space and then Newton proved that there is a direction, a vector, pointing to the periapsis, the point on the orbit closest to the attracting body. This vector was rediscovered many times and has born the names of many mathematicians - Laplace, Runge, Lenz et al. This vector is an integral of motion. As we know from Noether's work [38] integrals and symmetries are closely related and so there are additional symmetries. Also this extra integral implies that collision orbits are collinear which leads to the realization that the collision singularity was removable by the process which has become known as regularization.

Regularization was started in 2 dimensions by Goursat [19], then continued by Levi-Civita [27] using complex variable techniques and by Kustaanheimo-Stiefel using quaternions for 3 and 4 dimensions [26].

But regularization for the general $n$-dimensional problem uses the stereographic projection of the sphere onto a coordinate plane. Indeed the physicist Fock [18] exploited this projection in his investigation of the spectrum of the hydrogen atom from a quantum mechanical point of view. Moser [36] uses this projection to regularize the Kepler problem in $n$ dimensions. Although there are many important contributions (see, for instance [1, 20, 22]), for us the work of Moser [36] explains the additional integral, the extra symmetry, and the removable collision singularity while providing coordinates well adapted to our perturbation analysis.

So we start by recalling the regularization of the Kepler problem in $n$ dimensions due to Moser [36]. We follow the presentation in [23]. First of all let us consider the usual rectangular coordinates in the $n$-dimensional space, say $(q, p)=\left(q_{1}, \ldots, q_{n}, p_{1}, \ldots, p_{n}\right)$, and introduce the negative energy set $\Sigma_{-}=\left\{(q, p) \in T_{0} \mathbb{R}^{n} \mid \mathcal{H}(q, p)<0\right\}$, where $T_{0} \mathbb{R}^{n}=\left(\mathbb{R}^{n} \backslash\{0\}\right) \times \mathbb{R}^{n}$ and $\mathcal{H}$ refers to the Hamiltonian of the Kepler problem, i.e.,

$$
\mathcal{H}(q, p)=\frac{1}{2}|p|^{2}-\frac{\mu}{|q|},
$$

where || stands for the usual Euclidean norm and $\mu>0$ is the standard mass parameter of the Kepler problem.

We also define the tangent bundle of the $n$-sphere by $T \mathcal{S}^{n}=\left\{(x, y) \in T \mathbb{R}^{n+1}=\mathbb{R}^{n+1} \times \mathbb{R}^{n+1} \mid\right.$ $|x|=1, x \cdot y=0\}$ and $T^{+} \mathcal{S}^{n}=\left\{(x, y) \in T \mathcal{S}^{n} \mid y \neq 0\right\}$ with the operator $\cdot$ being the dot product.

If $\mathrm{np}$ represents the north pole of $\mathcal{S}^{n}$, i.e., $\mathrm{np}=x=(0, \ldots, 0,1)$ (with $x \in \mathbb{R}^{n+1}$ ) we introduce the Moser map [36] by

$$
\Phi_{\mathrm{M}}: T^{+}\left(\mathcal{S}^{n} \backslash\{\mathrm{np}\}\right) \rightarrow T_{0} \mathbb{R}^{n}:(x, y) \mapsto(q, p)
$$

where

$$
q_{i}=-\frac{1}{k}\left(y_{i}+x_{i} y_{n+1}-y_{i} x_{n+1}\right), \quad p_{i}=\frac{k x_{i}}{1-x_{n+1}},
$$

for $i=1, \ldots, n$, where $k$ is the positive parameter satisfying $h=-k^{2} / 2$ for a fixed negative value of the energy $\mathcal{H}=h$.

The inverse of $\Phi_{\mathrm{M}}$ is

$$
\Phi_{\mathrm{M}}^{-1}: \Sigma_{-} \subseteq T_{0} \mathbb{R}^{n} \rightarrow T^{+} \mathcal{S}^{n}:(q, p) \mapsto(x, y)
$$

REGULAR AND CHAOTIC DYNAMICS Vol. 00 No. $0 \quad 0000$ 
where

$$
\begin{aligned}
& x=\left(x_{1}, \ldots, x_{n}, x_{n+1}\right)=\left(\frac{2 k}{|p|^{2}+k^{2}} p, \frac{|p|^{2}-k^{2}}{|p|^{2}+k^{2}}\right), \\
& y=\left(y_{1}, \ldots, y_{n}, y_{n+1}\right)=\left(-\frac{1}{2 k}\left(\left(|p|^{2}+k^{2}\right) q-2(q \cdot p) p\right),-q \cdot p\right) .
\end{aligned}
$$

Moser regularization is accomplished by taking a new time, say $s$, introduced through

$$
\frac{d s}{d t}=\frac{k}{|q|} \text {. }
$$

Hence, the Kepler Hamiltonian must be scaled accordingly. Following Cushman [10] we define a new Hamiltonian, specifically,

$$
\tilde{\mathcal{H}}(q, p)=\frac{|q|}{k}\left(\mathcal{H}(q, p)+\frac{k^{2}}{2}\right)+\frac{\mu}{k}=\frac{|q|}{2 k}\left(|p|^{2}+k^{2}\right) .
$$

Now we restrict $q$ and $p$ to lie in the level set $\tilde{\mathcal{H}}^{-1}(\mu / k)$. It corresponds to the level set $\mathcal{H}^{-1}\left(-k^{2} / 2\right)$.

Applying Moser map (2.2) and taking into account that $x \cdot y=0$ and $|x|=1$, Hamiltonian (2.6) becomes

$$
\mathcal{H}^{*}(x, y)=|y|,
$$

which is the regularized Keplerian Hamiltonian defined on $T^{+} \mathcal{S}^{n}$. The flow of the Hamiltonian vector field $X_{\mathcal{H}^{*}}$ is the geodesic flow on $T^{+} \mathcal{S}^{n}, \mathcal{H}^{*}$ is smooth on $T^{+}\left(\mathcal{S}^{n} \backslash\{\mathrm{np}\}\right)$ and can be extended smoothly to the whole $T^{+} \mathcal{S}^{n}$.

Transformation (2.4) is symplectic when restricting the standard symplectic form $\sum_{i=1}^{n+1} d x_{i} \wedge d y_{i}$ on $T \mathbb{R}^{n+1}$ to $T^{+}\left(\mathcal{S}^{n} \backslash\{\mathrm{np}\}\right)$.

Rectilinear (i.e., collision) orbits of the Kepler problem correspond to the geodesic circles through the north pole np. Moreover, collision states given by $q=0$ and $|p|=\infty$ are transformed to $x=\mathrm{np}$ and $y=\left(y_{1}, \ldots, y_{n}, 0\right)$ with $|y|=\mu / k$.

Moser regularization works due to Hamilton's Theorem which states that the velocity vector associated with any non-degenerate Keplerian orbit moves along a circle, see for instance [34].

\section{MOSER REGULARIZATION IN TERMS OF DELAUNAY-LIKE COORDINATES}

\subsection{Lack of Analyticity on the Collision Manifold}

We start by considering the Kepler problem in two dimensions attaching to it a small smooth perturbation, see for instance [32]. As a prototype we choose the perturbation to be the radial distance. Thus, our initial Hamiltonian reads as

$$
\mathcal{H}_{\varepsilon}=\mathcal{H}+\varepsilon \mathcal{P}=\frac{1}{2}\left(R^{2}+\frac{\Theta^{2}}{r^{2}}\right)-\frac{\mu}{r}+\varepsilon r
$$

in the well known polar-symplectic set of coordinates $(r, \vartheta, R, \Theta)$, where $r$ and $\vartheta$ are the polar coordinates while $R$ and $\Theta$ denote their respective momenta. In particular, $R$ stands for the linear momentum in the $r$ direction and $\Theta$ represents the angular momentum, see [32]. The constant $\varepsilon>0$ is considered as a small parameter. It is clear that $\mathcal{H}_{\varepsilon}$ is analytic with respect to the four variables provided we avoid collisions.

At this point planar Delaunay coordinates $(\ell, g, L, G)$ are introduced where $\ell$ is the mean anomaly, $g$ the argument of perigee, $L$ the action conjugate to $\ell$ and $G=\Theta$. Hamiltonian (3.1) is transformed into

$$
\mathcal{H}_{\varepsilon}=-\frac{\mu^{2}}{2 L^{2}}+\varepsilon r,
$$


where $r$ cannot be written in terms of Delaunay elements explicitly due to the transcendental form that $r$ depends on. More specifically one has

$$
r=\alpha(1-e \cos E), \quad \text { where } \alpha=\frac{L^{2}}{\mu}, \quad e^{2}=1-\frac{G^{2}}{L^{2}},
$$

and $E$ denotes the eccentric anomaly that depends on the Delaunay coordinates $\ell, L$ and $G$ through the Kepler equation, that is,

$$
\ell=E-e \sin E=m t,
$$

where $m=\mu^{2} / L^{3}$ designates the mean motion and $t$ is the time variable.

Since it is impossible to put $E$ in terms of the Delaunay elements explicitly by using elementary functions, we cannot express $r$ as a function of this set, although we keep in mind that $r$ depends on $\ell, L$ and $G$.

Focusing on the perturbation $r$ written in Delaunay variables $r=r(\ell, L, G)$ a crucial point is that $r$ is not analytic with respect to $\ell$ when $G=0$, that is, at collisions. Indeed when $G=0$ one has $e=1$ and $r=\alpha(1-\cos E)$. Hence, the Kepler equation reads

$$
\ell=E-\sin E=\frac{1}{6} E^{3}+O\left(E^{5}\right),
$$

hence,

$$
E=6^{1 / 3} \ell^{1 / 3}+O(\ell)
$$

from where we deduce that $r$ in terms of $\ell$ and $L$ is given by

$$
r=\frac{3^{2 / 3} L^{2}}{2^{1 / 3} \mu} \ell^{2 / 3}+O(\ell) .
$$

Therefore, $r$ is not differentiable with respect to $\ell$ at $\ell=0$ when $G=0$. However, we notice that $r$ is analytic with respect to $E$ at collisions and everywhere else (the same is true with respect to the other coordinates).

The above paragraph highlights the convenience of rescaling the Hamiltonian changing the time in order to be able to consider motions of rectilinear type in a perturbed Kepler problem, thus discarding the use of Ligon-Schaaf regularization, excepting possible situations where the perturbation is analytic with respect to Delaunay coordinates, for instance in the case where the perturbation is a Fourier series in the mean anomaly.

\subsection{Moser and Spatial Delaunay-Like Coordinates}

Now we fix $n=3$ and want to establish the relationship between Delaunay and Moser coordinates. As a first step we need to complete the set of planar Delaunay coordinates adding the angle $\nu$ and its conjugate action $N$, where $\nu$ stands for the argument of the node and $N$ the projection of the angular momentum vector into the vertical axis while $G$, the conjugate action of $g$, represents the length of the angular momentum vector. We note that in the planar case $G$ can be negative or zero whereas in the spatial case it is always non negative. The reader can see more details on Delaunay variables in [5].

After fixing the passage through the perigee at the initial time, applying the Kepler equation and taking into account the relationship between $m$ and $\alpha$, that is, $\mu=m^{2} \alpha^{3}$, we get

$$
\frac{d E}{d t}=\frac{m}{1-e \cos E}=\frac{m \alpha}{r},
$$

where we have applied that $r=|q|=\alpha(1-e \cos E)$. Since $L=m \alpha^{2}=\sqrt{\mu \alpha}$ and we fix an energy level $h=-k^{2} / 2=-\mu^{2} /\left(2 L^{2}\right)$ we end up with

$$
\frac{d E}{d t}=\frac{\mu}{L r}=\frac{k}{r}=\frac{d s}{d t} .
$$

Thus we notice that the new time $s$ coincides essentially with the eccentric anomaly $E$. 
It is convenient to introduce a change of coordinates so that the eccentric anomaly gets transformed into a canonical variable, replacing the role played by $\ell$ as the fast angle. This transformation was firstly given by Levi-Civita [27]. It has been applied by other authors, see for instance [47] and more recently [17]. Starting from the symplectic set of polar-nodal coordinates $(r, \vartheta, \nu, R, \Theta, N)$ we introduce a new set given by $\left(E, \phi, \psi, P_{E}, \Phi, \Psi\right)$. The transformation is built through a generating function which is written in terms of the "old" coordinates, $r, \vartheta$ and $\nu$ and the "new" momenta $P_{E}, \Phi$ and $\Psi$. See the details in $[17,47]$. Explicitly one has

$$
\begin{aligned}
r & =\frac{1}{\sqrt{2 S}}\left(P_{E}-\sqrt{P_{E}^{2}-\Phi^{2}} \cos E\right), \\
\vartheta & =\phi-\tan ^{-1}\left(\frac{\Phi \sin E}{\sqrt{P_{E}^{2}-\Phi^{2}}-P_{E} \cos E}\right), \\
\nu & =\psi \\
R & =\frac{\sqrt{2 S} \sqrt{P_{E}^{2}-\Phi^{2}} \sin E}{P_{E}-\sqrt{P_{E}^{2}-\Phi^{2}} \cos E} \\
\Theta & =\Phi \\
N & =\Psi .
\end{aligned}
$$

In (3.3) $S$ plays the role of a parameter that has to be chosen adequately.

The change (3.3) converts Hamiltonian $\mathcal{H}$ in (3.1) into

$$
\mathcal{H}=-S-\frac{\mu-\sqrt{2 S} P_{E}}{r} .
$$

The parameter $S$ is chosen to be minus the energy of the Kepler problem. In terms of the constant $k$ or the action $L$ we readily get that

$$
S=\frac{k^{2}}{2}=\frac{\mu^{2}}{2 L^{2}}
$$

Therefore the phase flow determined by $\mathcal{H}$ of $(3.4)$ on the manifold $\mathcal{H}=-S$ in the chart $\left(E, \phi, \psi, P_{E}, \Phi, \Psi, t\right)$ is in 1:1 correspondence with the phase flow determined by $\mathcal{H}^{*}$ defined as

$$
\mathcal{H}^{*}=\frac{r}{\sqrt{2 S}}(\mathcal{H}+S)+\frac{\mu}{\sqrt{2 S}}=P_{E}
$$

on the manifold $P_{E}=L$ in the chart $\left(E, \phi, \psi, P_{E}, \Phi, \Psi, s\right)$, since $\mu / \sqrt{2 S}=L$. Notice that the factor $r / \sqrt{2 S}$ is equivalent to $|q| / k$.

The relation between the times $t$ and $s$ is given by

$$
t=s-\frac{1}{2 S} \sqrt{P_{E}^{2}-\Phi^{2}} \sin E
$$

From now on we identify $\phi=g, \psi=\nu, \Phi=G$ and $\Psi=N$. We stress that $G=\Theta \geq 0$ and $L$ is no longer a variable but a parameter related with $S$ and $k$ by means of $(3.5)$. The set $(E, g, \nu$, $\left.P_{E}, G, N\right)$ is the one that we call Delaunay-like coordinates. They are also called Delaunay-similar or Delaunay-Scheifele coordinates.

In order to express Moser coordinates in terms of Delaunay-like ones we start by putting the polar symplectic coordinates introduced in Section 3.1 in terms of rectangular planar coordinates $(u, v, U, V)$ by means of

$$
u=r \cos \vartheta, \quad U=R \cos \vartheta-\frac{\Theta}{r} \sin \vartheta, \quad v=r \sin \vartheta, \quad V=R \sin \vartheta+\frac{\Theta}{r} \cos \vartheta .
$$


At this point we compose the transformation (3.6) with the rectangular coordinates $(q, p)$ in the three-dimensional space through

$$
\begin{aligned}
& q_{1}=u \cos \nu-\bar{c} v \sin \nu, \quad q_{2}=u \sin \nu+\bar{c} v \cos \nu, \quad q_{3}=\bar{s} v, \\
& p_{1}=U \cos \nu-\bar{c} V \sin \nu, \quad p_{2}=U \sin \nu+\bar{c} V \cos \nu, \quad p_{3}=\bar{s} V,
\end{aligned}
$$

where $\bar{s}=\sin I=\sqrt{1-N^{2} / \Theta^{2}}, \bar{c}=\cos I=N / \Theta$ and $I$ is the inclination angle between the orbital and the equatorial planes.

After plugging (3.6) in (3.7), applying thereafter transformation (3.3) and simplifying the intermediate expressions we end up with

$$
\begin{aligned}
q_{1} & =\frac{L}{\mu} P_{E}(((\cos E-\tilde{e}) \cos g-\tilde{\eta} \sin E \sin g) \cos \nu-\bar{c}(\tilde{\eta} \sin E \cos g+(\cos E-\tilde{e}) \sin g) \sin \nu), \\
q_{2} & =\frac{L}{\mu} P_{E}(\bar{c}(\tilde{\eta} \sin E \cos g+(\cos E-\tilde{e}) \sin g) \cos \nu+((\cos E-\tilde{e}) \cos g-\tilde{\eta} \sin E \sin g) \sin \nu), \\
q_{3} & =\frac{L}{\mu} P_{E} \bar{s}(\tilde{\eta} \sin E \cos g+(\cos E-\tilde{e}) \sin g), \\
p_{1} & =\frac{\mu}{L(1-\tilde{e} \cos E)}(-(\sin E \cos g+\tilde{\eta} \cos E \sin g) \cos \nu+\bar{c}(\sin E \sin g-\tilde{\eta} \cos E \cos g) \sin \nu), \\
p_{2} & =\frac{\mu}{L(1-\tilde{e} \cos E)}(\bar{c}(\tilde{\eta} \cos E \cos g-\sin E \sin g) \cos \nu-(\sin E \cos g+\tilde{\eta} \cos E \sin g) \sin \nu), \\
p_{3} & =\frac{\mu}{L(1-\tilde{e} \cos E)} \bar{s}(\tilde{\eta} \cos E \cos g-\sin E \sin g),
\end{aligned}
$$

where we have introduced $\tilde{\eta}=G / P_{E}$ and $\tilde{e}=\sqrt{1-\tilde{\eta}^{2}}$.

We link Moser coordinates of the spatial Kepler problem with Delaunay-like ones.

Proposition 1. When $n=3$ Moser coordinates (2.5) are written in terms of spatial Delaunay-like coordinates by means of the following formulae:

$$
\begin{aligned}
& x_{1}=-\tilde{\eta} \cos E(\sin g \cos \nu+\bar{c} \cos g \sin \nu)-\sin E(\cos g \cos \nu-\bar{c} \sin g \sin \nu), \\
& y_{1}=P_{E} \tilde{\eta} \sin E(\sin g \cos \nu+\bar{c} \cos g \sin \nu)-P_{E} \cos E(\cos g \cos \nu-\bar{c} \sin g \sin \nu), \\
& x_{2}=-\sin E(\bar{c} \sin g \cos \nu+\cos g \sin \nu)+\tilde{\eta} \cos E(\bar{c} \cos g \cos \nu-\sin g \sin \nu), \\
& y_{2}=-P_{E} \cos E(\bar{c} \sin g \cos \nu+\cos g \sin \nu)-P_{E} \tilde{\eta} \sin E(\bar{c} \cos g \cos \nu-\sin g \sin \nu), \\
& x_{3}=\bar{s}(\tilde{\eta} \cos E \cos g-\sin E \sin g), \\
& y_{3}=-P_{E} \bar{s}(\tilde{\eta} \sin E \cos g+\cos E \sin g), \\
& x_{4}=\tilde{e} \cos E \\
& y_{4}=-P_{E} \tilde{e} \sin E .
\end{aligned}
$$

Proof (of Proposition 1). First we apply (3.8) to (2.5), assuming $n=3$. Next we simplify all the resulting formulae arriving at (3.9).

These formulae make sense in the domain where Delaunay-like coordinates are well defined, i.e., where the momenta satisfy $|N|<G<P_{E}$, thus at the beginning one should exclude rectilinear, circular and equatorial motions, although the above expressions can be extended to circular motions 
$\left(G=P_{E}\right.$ in Delaunay-like coordinates) and to equatorial ones $(|N|=G)$. We shall go back to this in Section 4.2.

The application of $(3.9)$ to $\mathcal{H}^{*}$ of $(2.7)$ yields the Hamiltonian

$$
\mathcal{H}^{*}=P_{E} \text {. }
$$

\section{NORMALIZING A SPATIAL PERTURBED KEPLERIAN SYSTEM}

\subsection{Normalization in Delaunay-Like Coordinates}

Let us consider the perturbed Hamiltonian function

$$
\mathcal{H}_{\varepsilon}(q, p)=\mathcal{H}(q, p)+\varepsilon \mathcal{P}(q, p ; \varepsilon)
$$

where $\mathcal{H}$ is the Hamiltonian of the Kepler problem given in (2.1),

$$
\mathcal{P}(q, p ; \varepsilon)=\mathcal{P}_{1}(q, p)+\varepsilon \mathcal{P}_{2}(q, p)+\varepsilon^{2} \mathcal{P}_{3}(q, p)+\cdots,
$$

is a regular perturbation and $\varepsilon>0$ designates a small parameter. We keep $n=3$ in this section.

Our plan is to average (i.e., normalize) $\mathcal{H}_{\varepsilon}$ with respect to the solutions of the Keplerian part but in the framework of Moser regularization. As in the unperturbed case we fix an energy value, say $h<0$, thus we work on the energy level $\mathcal{H}_{\varepsilon}=h$ and define $k>0$ such that $h=-k^{2} / 2$. Then we introduce the Hamiltonian

$$
\tilde{\mathcal{H}}_{\varepsilon}(q, p)=\frac{|q|}{k}\left(\mathcal{H}_{\varepsilon}(q, p)+\frac{k^{2}}{2}\right)+\frac{\mu}{k} .
$$

We restrict $q, p$ to lie in the level set $\tilde{\mathcal{H}}_{\varepsilon}^{-1}(\mu / k)$, which corresponds to the level set $\mathcal{H}_{\varepsilon}^{-1}\left(-k^{2} / 2\right)$. We also take into account that $\mathcal{H}_{\varepsilon}$ is negative, thus perturbations of bounded Keplerian solutions are treated.

Notice that the factor $|q| / k$ in (4.2) after applying (2.3) reads as

$$
f(x, y)=\frac{1}{k^{2}} \sqrt{\left(y_{1}+y_{4} x_{1}-y_{1} x_{4}\right)^{2}+\left(y_{2}+y_{4} x_{2}-y_{2} x_{4}\right)^{2}+\left(y_{3}+y_{4} x_{3}-y_{3} x_{4}\right)^{2}} .
$$

After the scaling of time $\mathcal{P}(q, p ; \varepsilon)$ gets transformed through $(2.3)$ into the term $\mathcal{P}^{*}(x, y ; \varepsilon)$ $=f(x, y) \mathcal{P}(x, y ; \varepsilon)$. Hence, Hamiltonian (4.2) becomes

$$
\mathcal{H}_{\varepsilon}^{*}(x, y)=|y|+\varepsilon \mathcal{P}^{*}(x, y ; \varepsilon),
$$

which is the regularized Hamiltonian corresponding to $\mathcal{H}_{\varepsilon}$ defined on $T^{+} \mathcal{S}^{3}$. Besides, the flow of the Hamiltonian vector field $X_{\mathcal{H}_{\varepsilon}^{*}}$ is the geodesic flow on $T^{+} \mathcal{S}^{3}$ and $\mathcal{H}_{\varepsilon}^{*}$ is a smooth function on $T^{+}\left(\mathcal{S}^{3} \backslash\{\mathrm{np}\}\right)$ that may be extended smoothly to the tangent bundle of $\mathcal{S}^{3}$ minus its zero section, i.e., $T^{+} \mathcal{S}^{3}$.

In our context normalizing Hamiltonian $\mathcal{H}_{\varepsilon}^{*}$ means to perform a symplectic change of coordinates, formally in powers of $\varepsilon$, up to a finite order, say $p$, so that $\mathcal{H}_{\varepsilon}^{*}$ gets transformed into

$$
|y|+\varepsilon \mathcal{H}_{1}^{*}+\varepsilon^{2} \mathcal{H}_{2}^{*}+\varepsilon^{3} \mathcal{H}_{3}^{*}+\ldots+\varepsilon^{p} \mathcal{H}_{p}^{*}+O\left(\varepsilon^{p+1}\right),
$$

and each $\mathcal{H}_{j}^{*}$ (with $1 \leq j \leq p$ ) commutes with $|y|$. The same idea applies to any dimension $n$ of the Kepler problem.

Proposition 2. The application of the map (3.9) to (4.3) produces a Hamiltonian in Delaunaylike coordinates that is normalized by averaging with respect to the eccentric anomaly to obtain the corresponding Hamiltonian in normal form.

Proof (of Proposition 2). We write down Hamiltonian $\mathcal{H}_{\varepsilon}^{*}$ in terms of Delaunay-like coordinates. First notice that by virtue of (3.9) we get $|y|=P_{E}$. Besides, since $|q|=r$, the factor $f(x, y)$ becomes $f(r)=r / k$, thus one gets $\mathcal{P}^{*}=(r / k) \mathcal{P}$ where $r$ is put in terms of $E, P_{E}$ and $G$ and the parameter $L$. If $\mathcal{P}$ is smooth with respect to $x$ and $y$ then $\mathcal{P}^{*}$ is smooth with respect to $E, P_{E}, g, \nu, \tilde{e}, \tilde{\eta}, \bar{c}$, $\bar{s}$. In particular the dependence with respect to $E$ occurs through terms of the form $\sin (j E)$ and $\cos (j E)$ with $j$ a non-negative integer. The smooth character is maintained even for collision states, 
that is, when $E=0(\bmod 2 \pi)$ and $G=N=0$. When $\mathcal{P}$ is a polynomial in $q$ and $p$ and linear in $p$, then $\mathcal{P}^{*}$ is a finite Fourier series in the angles $E, g$ and $\nu$ whose coefficients depend smoothly on $P_{E}, \bar{c}, \bar{s}, \tilde{e}$ and $\tilde{\eta}$. It is possible as well that $\mathcal{P}$ contain terms with $|q|$ in its denominators.

Summarizing one is confronted with a perturbation problem with Hamiltonian

$$
\mathcal{H}_{\varepsilon}^{*}=P_{E}+\varepsilon \mathcal{P}^{*}\left(E, g, \nu, P_{E}, G, N ; \varepsilon, L\right)=P_{E}+\varepsilon \mathcal{H}_{1}^{*}+\varepsilon^{2} \mathcal{H}_{2}^{*}+\cdots,
$$

where we identify $\mathcal{H}_{0}^{*}$ with $P_{E}, \mathcal{H}_{k}^{*}$ with $\mathcal{P}_{k}^{*}$, and $\mathcal{P}_{k}^{*}=(r / k) \mathcal{P}_{k}$ for $k=1,2, \ldots$

An important feature of our regularization process is that $L=\mu / k$ and as the perturbation depends on $E$ all what we need to do is to normalize $\mathcal{H}_{\varepsilon}^{*}$ with respect to it, using for instance a procedure based on Lie transformations [13,32], ending up with a Hamiltonian function independent of $E$ up to a certain order, thence $P_{E}$ becomes a constant of motion, after truncating higher-order terms. Finally, in order to recover the flow that corresponds to the Hamiltonian system prior to regularization, one simply sets $P_{E}=L$ in the normalized Hamiltonian.

Remark 1. When we do not consider near-collision motions the application of Proposition 2, up to first order in the small parameter, is equivalent to averaging with respect to the mean anomaly in Delaunay elements, without regularizing. The reason is that the average of a certain function $F$ with respect to $\ell$ is obtained as

$$
\bar{F}=\frac{1}{2 \pi} \int_{0}^{2 \pi} F d \ell=\frac{1}{2 \pi} \int_{0}^{2 \pi}(r / \alpha) F d E
$$

whereas applying the above procedure we get

$$
\tilde{F}=\frac{1}{2 \pi} \int_{0}^{2 \pi}(r / k) F^{\&} d E
$$

where $F^{\&}$ refers to $F$ written in Delaunay-like coordinates. Hence, on the manifold $P_{E}=L$ one gets $\tilde{F}=\left(L^{3} / \mu^{2}\right) \bar{F}$ (after setting $P_{E}=L$ in $\tilde{F}$ ). Note however that since we have regularized the collisions $r=0$ and $\ell$ is not present in $(4.4), \mathcal{H}_{\varepsilon}^{*}$ can be extended smoothly to the collision set (provided $\mathcal{P}^{*}$ is smooth when $r=0$ ) making our method valid for near-rectilinear motions. More precisely, the solutions that are of rectilinear character can be dealt with using a set of canonical variables defined from the Delaunay ones, see [49]. On the other hand circular and equatorial motions can be analyzed using adequate linear combinations of the Delaunay variables, see for instance [24].

Remark 2. Sometimes it is convenient to use the true anomaly instead of the eccentric anomaly in order to achieve the normalization of a perturbed Keplerian Hamiltonian when $n=2,3$. This allows one to enlarge the set of functions where Delaunay normalization is applied up to a large extent. An account of these techniques is given in [14, 39, 40].

\subsection{Normalization in $\mathcal{S}^{2} \times \mathcal{S}^{2}$}

As we have seen in the previous subsection, the regularized Hamiltonian is independent of $\ell$ and its conjugate action $L$ plays the role of a constant. Thus $\mathcal{H}_{\varepsilon}^{*}$ introduced in (4.4) can be expressed as a function of the eccentric anomaly and the invariants that define the symplectic manifold $\mathcal{S}^{2} \times \mathcal{S}^{2}$ (see [36]) where the reduced Hamiltonian lies. In fact this is the space resulting after normalizing to remove the dependence with respect to $E$.

To achieve the normalization process one introduces the so called Keplerian invariants, which is a collection of six variables given by $a=\left(a_{1}, a_{2}, a_{3}\right)$ and $b=\left(b_{1}, b_{2}, b_{3}\right)$. On the energy level $\tilde{\mathcal{H}}_{\varepsilon}^{-1}(\mu / k)$, $a$ and $b$ satisfy the constraints $|a|=|b|=P_{E}$. These coordinates parameterize the reduced space $\mathcal{S}^{2} \times \mathcal{S}^{2}$ and are defined in terms of the rectangular coordinates as follows:

$$
a=\mathbf{G}+L A, \quad b=\mathbf{G}-L A
$$

REGULAR AND CHAOTIC DYNAMICS Vol. 00 No. $0 \quad 0000$ 
where $\mathbf{G}=q \times p$ is the angular momentum vector (by $\times$ we designate the cross product in $\mathbb{R}^{3}$ ), and $A$ is the Laplace-Runge-Lenz vector given by

$$
A=\frac{1}{\mu}(p \times \mathbf{G})-\frac{q}{|q|} .
$$

The invariants $a_{i}$ and $b_{i}$ belong to the interval $\left[-P_{E}, P_{E}\right]$.

The explicit expressions of $a$ and $b$ in terms of Delaunay variables are found in Cushman [8], see also [40]. We modify them slightly to adapt the formulae to the case of Delaunay-like variables. We introduce:

$$
\begin{aligned}
& a_{1}=\bar{s} G \sin \nu+P_{E} \tilde{e}(\cos g \cos \nu-\bar{c} \sin g \sin \nu), \\
& a_{2}=-\bar{s} G \cos \nu+P_{E} \tilde{e}(\cos g \sin \nu+\bar{c} \sin g \cos \nu), \\
& a_{3}=\bar{c} G+P_{E} \bar{s} \tilde{e} \sin g, \\
& b_{1}=\bar{s} G \sin \nu-P_{E} \tilde{e}(\cos g \cos \nu-\bar{c} \sin g \sin \nu), \\
& b_{2}=-\bar{s} G \cos \nu-P_{E} \tilde{e}(\cos g \sin \nu+\bar{c} \sin g \cos \nu), \\
& b_{3}=\bar{c} G-P_{E} \bar{s} \tilde{e} \sin g .
\end{aligned}
$$

Note that with the use of the invariants we avoid the problems caused by the indetermination of Delaunay coordinates. In particular, from the identity $2 G=|a+b|$, whence $G=0$ if and only if $b=-a$. So the subset of $\mathcal{S}^{2} \times \mathcal{S}^{2}$ defined by

$$
\mathcal{R}=\left\{(a,-a) \in \mathbb{R}^{6}|| a \mid=P_{E}\right\}
$$

is a two-dimensional set diffeomorphic to $\mathcal{S}^{2}$ consisting of rectilinear trajectories. Note that the collision state is also represented by the set $\mathcal{R}$.

On the one hand, circular trajectories satisfy the condition $G=P_{E}$ in the spatial Delaunay elements, and in terms of $a$ and $b$ this implies that $a=b$. Thus, circular motions define the following two-dimensional set diffeomorphic to $\mathcal{S}^{2}$ :

$$
\mathcal{C}=\left\{(a, a) \in \mathbb{R}^{6}|| a \mid=P_{E}\right\} .
$$

On the other hand equatorial trajectories satisfy $G=|N|$ and are given in terms of the invariants by the two-dimensional set

$$
\mathcal{E}=\left\{(a, b) \in \mathbb{R}^{6}|| a \mid=P_{E}, b_{1}=-a_{1}, b_{2}=-a_{2}, b_{3}=a_{3}\right\},
$$

which is again diffeomorphic to $\mathcal{S}^{2}$.

The introduction of the Keplerian invariants $a$ and $b$ extends the use of Delaunay and Delaunaylike coordinates, as we can include equatorial, circular and rectilinear solutions [51]. The other points on $\mathcal{S}^{2} \times \mathcal{S}^{2}$ correspond to elliptic orbits of the spatial Kepler problem.

Regarding the planar case we can use the invariants $a=\left(a_{1}, a_{2}, a_{3}\right)$ with $a_{1}^{2}+a_{2}^{2}+a_{3}^{2}=P_{E}^{2}$ introduced in [51], which define the reduced space $\mathcal{S}^{2}$ where the perturbed Hamiltonian lies. For a generalization to the $n$-dimensional Kepler problem, one introduces a set of invariants related to the angular momentum vector and the Laplace-Runge-Lenz vector in $n$ dimensions, see details in [23]. We shall study this latter situation in Section 5.

We are ready to state our next result. 
Proposition 3. Moser coordinates (2.4) for $n=3$ are written in terms of the Keplerian invariants (4.5), E and $P_{E}$ by means of the map:

$$
\begin{aligned}
& x_{1}=\frac{1}{P_{E} \kappa}\left(P_{E}\left(b_{1}-a_{1}\right) \sin E+\left(a_{3} b_{2}-a_{2} b_{3}\right) \cos E\right), \\
& y_{1}=\frac{1}{\kappa}\left(\left(a_{2} b_{3}-a_{3} b_{2}\right) \sin E+P_{E}\left(b_{1}-a_{1}\right) \cos E\right), \\
& x_{2}=\frac{1}{P_{E} \kappa}\left(P_{E}\left(b_{2}-a_{2}\right) \sin E+\left(a_{1} b_{3}-a_{3} b_{1}\right) \cos E\right), \\
& y_{2}=\frac{1}{\kappa}\left(\left(a_{3} b_{1}-a_{1} b_{3}\right) \sin E+P_{E}\left(b_{2}-a_{2}\right) \cos E\right), \\
& x_{3}=\frac{1}{P_{E} \kappa}\left(P_{E}\left(b_{3}-a_{3}\right) \sin E+\left(a_{2} b_{1}-a_{1} b_{2}\right) \cos E\right), \\
& y_{3}=\frac{1}{\kappa}\left(\left(a_{1} b_{2}-a_{2} b_{1}\right) \sin E+P_{E}\left(b_{3}-a_{3}\right) \cos E\right), \\
& x_{4}=\frac{\kappa}{2 P_{E}} \cos E \\
& y_{4}=-\frac{\kappa}{2} \sin E
\end{aligned}
$$

where

$$
\kappa=2 P_{E} \tilde{e}=\sqrt{2\left(P_{E}^{2}-a \cdot b\right)} .
$$

The inverse of the map (4.8) is given by:

$$
\begin{aligned}
& a_{1}=x_{1} y_{4}-x_{4} y_{1}+x_{2} y_{3}-x_{3} y_{2}, \quad b_{1}=x_{4} y_{1}-x_{1} y_{4}+x_{2} y_{3}-x_{3} y_{2}, \\
& a_{2}=x_{3} y_{1}-x_{1} y_{3}+x_{2} y_{4}-x_{4} y_{2}, \quad b_{2}=x_{3} y_{1}-x_{1} y_{3}+x_{4} y_{2}-x_{2} y_{4}, \\
& a_{3}=x_{1} y_{2}-x_{2} y_{1}+x_{3} y_{4}-x_{4} y_{3}, \quad b_{3}=x_{1} y_{2}-x_{2} y_{1}+x_{4} y_{3}-x_{3} y_{4} .
\end{aligned}
$$

Proof (of Proposition 3). The transformation (4.8) is obtained as follows. Starting from the relations (3.9) and putting later $\cos g, \sin g, \cos \nu, \sin \nu, \tilde{e}, \tilde{\eta}, \bar{c}$ and $\bar{s}$ in terms of $a$ and $b$ by means of the mapping (4.7), the transformation gives Moser coordinates as functions of the invariants $a, b$ and the canonical pair $E / P_{E}$, that is, the expressions of (4.8).

To get (4.9) the invariants $a$ and $b$ are written in terms of the rectangular coordinates $q$ and $p$ through (4.5). Next we compose the resulting expressions with the map $\Phi_{\mathrm{M}}$ and perform some simplifications, ending up with the formulae given in (4.9).

Special care must be taken when $\kappa=0$, that is, for circular trajectories, where Moser coordinates make sense although (4.8) does not apply in principle. We stress that circular motions are identified by the conditions

$$
x_{4}=y_{4}=0, \quad x_{1}^{2}+x_{2}^{2}+x_{3}^{2}=1, \quad x_{1} y_{1}+x_{2} y_{2}+x_{3} y_{3}=0, \quad y_{1}^{2}+y_{2}^{2}+y_{3}^{2}=P_{E}^{2} .
$$

REGULAR AND CHAOTIC DYNAMICS Vol. 00 No. $0 \quad 0000$ 
Formulae (4.8) allow us to obtain the explicit expressions of the rectangular coordinates in terms of the Keplerian invariants and the eccentric anomaly. Combining (2.3) with (4.8) we end up with

$$
\begin{aligned}
& q_{1}=\frac{L}{2 \mu \kappa}\left(2\left(a_{3} b_{2}-a_{2} b_{3}\right) \sin E+\left(a_{1}-b_{1}\right)\left(2 P_{E} \cos E-\kappa\right)\right), \\
& p_{1}=\frac{2 \mu}{L \kappa\left(\kappa \cos E-2 P_{E}\right)}\left(P_{E}\left(a_{1}-b_{1}\right) \sin E+\left(a_{2} b_{3}-a_{3} b_{2}\right) \cos E\right), \\
& q_{2}=\frac{L}{2 \mu \kappa}\left(2\left(a_{1} b_{3}-a_{3} b_{1}\right) \sin E+\left(a_{2}-b_{2}\right)\left(2 P_{E} \cos E-\kappa\right)\right), \\
& p_{2}=\frac{2 \mu}{L \kappa\left(\kappa \cos E-2 P_{E}\right)}\left(P_{E}\left(a_{2}-b_{2}\right) \sin E+\left(a_{3} b_{1}-a_{1} b_{3}\right) \cos E\right), \\
& q_{3}=\frac{L}{2 \mu \kappa}\left(2\left(a_{2} b_{1}-a_{1} b_{2}\right) \sin E+\left(a_{3}-b_{3}\right)\left(2 P_{E} \cos E-\kappa\right)\right), \\
& p_{3}=\frac{2 \mu}{L \kappa\left(\kappa \cos E-2 P_{E}\right)}\left(P_{E}\left(a_{3}-b_{3}\right) \sin E+\left(a_{1} b_{2}-a_{2} b_{1}\right) \cos E\right) .
\end{aligned}
$$

By means of (4.10) a Hamiltonian function like (4.1) is written in terms of the Keplerian invariants $a$ and $b$ and the angle-action pair $E / P_{E}$. So, the perturbation becomes a function of $E$, $P_{E}, a, b$, the energy-parameter $k, \mu$ and perhaps other external parameters, thence it is expressed as a function of the form $\mathcal{P}^{*}\left(a, b, E, P_{E} ; \mu, k\right)$. Notice that if the perturbation is linear in $p_{i}$ and polynomial in $q_{i}, \mathcal{P}^{*}$ is a finite Fourier series in $\cos E, \sin E$, because $\kappa \cos E-2 P_{E}=-2(\mu / L) r$ and the multiplication of $\mathcal{P}$ by $f(r)$ avoids the occurrence of $\kappa \cos E-2 P_{E}$ in the denominators of $\mathcal{P}^{*}$. have

We have to take into account the Poisson structure involving $a, b, E$ and $P_{E}$. Specifically we

$$
\begin{aligned}
& \left\{a_{1}, a_{2}\right\}=2 a_{3}, \quad\left\{a_{2}, a_{3}\right\}=2 a_{1}, \quad\left\{a_{3}, a_{1}\right\}=2 a_{2}, \\
& \left\{b_{1}, b_{2}\right\}=2 b_{3}, \quad\left\{b_{2}, b_{3}\right\}=2 b_{1}, \quad\left\{b_{3}, b_{1}\right\}=2 b_{2}, \\
& \left\{a_{i}, b_{j}\right\}=0, \\
& \left\{a_{i}, E\right\}=-\left\{b_{i}, E\right\}=\frac{\left(b_{i}-a_{i}\right) P_{E}}{P_{E}^{2}-a_{1} b_{1}-a_{2} b_{2}-a_{3} b_{3}}, \\
& \left\{a_{i}, P_{E}\right\}=\left\{b_{i}, P_{E}\right\}=0, \\
& \left\{E, P_{E}\right\}=1,
\end{aligned}
$$

see [51] for the brackets involving $a$ and $b$. In fact, the Poisson brackets of the $a_{i}$ correspond to the Lie algebra so(3) and the same is true for the Poisson brackets of the $b_{i}$. To obtain the brackets $\left\{a_{i}, E\right\},\left\{b_{i}, E\right\}$ we have put $a$ and $b$ in terms of Delaunay-like coordinates, have computed the corresponding Poisson brackets in this set and have returned to the expressions in the invariants $a, b$ and $P_{E}$.

We emphasize that the normalization process involving the invariants $a, b, P_{E}$ and $E$ has to be done in the framework of the constrained normal form theory $[10,12,50]$ because the constraints $|a|=|b|=P_{E}$ must be taken into consideration when carrying out the calculations.

All the ingredients are ready in order to perform the normalization of a given perturbed Hamiltonian system with the aim of converting $P_{E}$ into an integral of motion, up to a certain order in $\varepsilon$. One applies the Lie transformation to a certain order, using the Poisson structure given in (4.11) to compute the intermediate Hamiltonians needed in the calculation of the Lie triangle. As a final step the higher-order terms are dropped. The resulting Hamiltonian is a function of the invariants $a$ and $b$ and $P_{E}$ (apart from $L$ and other parameters), thus valid for all kind of bounded 
motions. The final step consists in setting $P_{E}=L$ so that we recover the flow corresponding with the Hamiltonian before regularizing. Notice that in the reduced space $\mathcal{S}^{2} \times \mathcal{S}^{2}$ the length of $a$ and $b$ is $L$ and $\tilde{e}=e, \tilde{\eta}=\eta$.

We end up with the following result.

Proposition 4. The regularized Hamiltonian function (4.2) is normalized with respect to the eccentric anomaly $E$ after applying to it the map (4.10). The procedure is carried out by means of the Lie transformation method taking into consideration that $\mathcal{H}^{*}=P_{E}$. The corresponding calculations for orders higher than one make use of the Poisson structure (4.11). If the perturbation $\mathcal{P}^{*}(x, y ; \varepsilon)$ in (4.3) is smooth on $T^{+} \mathcal{S}^{3}$, the normalization is performed in the whole $T^{+} \mathcal{S}^{3}$.

Proof (of Proposition 4). It follows from the preceding paragraphs.

When $\kappa=0$ the change (4.10) can still be applied to carry out an averaging obtaining the normalized Hamiltonian and the corresponding generating function. Then, if the final expressions after simplifications are singular for circular orbits, one should return to Moser coordinates applying (4.9) and putting also $P_{E}, \sin E, \cos E$ in terms of $x, y$ and check whether the terms are well defined for circular motions. Specifically one has to make the replacements $\kappa=2 \sqrt{|y|^{2} x_{4}^{2}+y_{4}^{2}}, P_{E}=|y|$ and from the last two formulae of (4.8)

$$
\cos E=\frac{|y| x_{4}}{\sqrt{|y|^{2} x_{4}^{2}+y_{4}^{2}}}, \quad \sin E=-\frac{y_{4}}{\sqrt{|y|^{2} x_{4}^{2}+y_{4}^{2}}} .
$$

One usually needs to simplify the resulting expressions by means of computer algebra techniques, basically taking into account the existing constraints for $x, y$. More precisely one starts by finding a Gröbner basis formed by the invariants and the related constraints, and apply to the formula one wants to express in terms of the invariants the multivariate division algorithm with respect to the Gröbner basis, see [48]. The remainder of the division is written in terms of the invariants, see more details in $[41,46]$. Thus, one checks whether the formulae in Moser coordinates are valid for circular motions, that is, they are smooth in these variables. On the other hand the transformation obtained above is not smooth for the momenta $p_{i}$ in the collision state $q=0$ because then $\sin E=0$, $\cos E=1, \kappa=2 P_{E}$ and $\kappa \cos E-2 P_{E}=0$. Nevertheless, if the terms in the perturbation $\mathcal{P}$ are linear in the momenta but they do not present other singularities, then $\mathcal{P}^{*}$ extends to a smooth function defined on the entire $T^{+} \mathcal{S}^{3}$ and one can perform the normalization applying (4.10). Analogously if $\mathcal{P}$ is smooth for circular motions the normalization can be achieved through the steps described above.

Remark 3. At this point it is reasonable to compare the procedure followed in this section with the one of van der Meer and Cushman [50] when $n=3$. Both approaches lead to the same results and one can relate the normalized Hamiltonians and their associated generating functions obtained with the two methods using the formulae (4.9), (4.8) and (4.12). On the one hand, the method of van der Meer and Cushman uses the fact that Hamiltonian (2.7) is a linear oscillator, thus its flow is periodic which allows for a splitting based on the normal form theory of Cushman [9]. On the other hand our result makes use of the fact that after applying (4.10), the action $L$ becomes a parameter and the normalization process is merely an average with respect to $E$. From a practical point of view, generically the normalization in Moser coordinates involves simpler expressions. This is the case if one proceeds carefully taking into account the constraints between $x$ and $y$ making use of Gröbner bases, avoiding besides the indetermination caused when $\kappa=0$. Then, one can apply (4.8) to write down the resulting normal form in terms of the Keplerian invariants $a, b$. Notice that since the Hamiltonian is normalized the final form of the Hamiltonian is independent of $E$ whereas $P_{E}$ becomes an (approximate) integral. If one decides to proceed in Moser coordinates, the following Poisson structure has to be taken into account when carrying out the computation of the intermediate Hamiltonians. For $1 \leq i, j \leq 4$ straightforward computations show that

$$
\left\{x_{i}, x_{j}\right\}=0, \quad\left\{y_{i}, y_{j}\right\}=x_{j} y_{i}-x_{i} y_{j}, \quad\left\{x_{i}, y_{i}\right\}=1-x_{i}^{2}, \quad\left\{x_{i}, y_{j}\right\}=-x_{i} x_{j}(i \neq j) .
$$


Going back to the example of Section 3.1, the perturbation $r$ given in Hamiltonian (3.1), but thought as a perturbation of the spatial Kepler problem, is obtained in terms of $a$ and $b$ after applying (4.10). One gets

$$
r=\frac{L}{2 \mu}\left(2 P_{E}-\kappa \cos E\right)
$$

therefore, taking into account that Hamiltonian $\mathcal{H}_{\varepsilon}$ has to be multiplied by $r / k$, the regularized Hamiltonian becomes

$$
\mathcal{H}_{\varepsilon}^{*}=P_{E}+\varepsilon \frac{L^{3}}{4 \mu^{3}}\left(2 P_{E}-\kappa \cos E\right)^{2},
$$

and its first-order average obtained after normalizing the perturbation over $E$ yields

$$
\mathcal{H}_{\varepsilon}^{*}=P_{E}+\varepsilon \frac{L^{3}}{4 \mu^{3}}\left(5 P_{E}^{2}-a_{1} b_{1}-a_{2} b_{2}-a_{3} b_{3}\right)+\mathcal{O}\left(\varepsilon^{2}\right) .
$$

Finally we have to set $P_{E}=L$ to deal with the normalized Hamiltonian in the right energy level, thus

$$
\mathcal{H}_{\varepsilon}^{*}=L+\varepsilon \frac{L^{3}}{4 \mu^{3}}\left(5 L^{2}-a_{1} b_{1}-a_{2} b_{2}-a_{3} b_{3}\right)+\mathcal{O}\left(\varepsilon^{2}\right) .
$$

The associated generating function is given by

$$
\mathcal{W}=-\varepsilon \frac{L^{3}}{8 \mu^{3}}\left(8 P_{E} \sqrt{2\left(P_{E}^{2}-a_{1} b_{1}-a_{2} b_{2}-a_{3} b_{3}\right)} \sin E+\left(a_{1} b_{1}+a_{2} b_{2}+a_{3} b_{3}-P_{E}^{2}\right) \sin (2 E)\right)
$$

The normal form $\mathcal{H}_{\varepsilon}^{*}$ and the generating function $\mathcal{W}$ are written in terms of Moser coordinates by applying (4.9). After some simplifications we end up with the expressions

$$
\mathcal{H}_{\varepsilon}^{*}=|y|+\varepsilon \frac{L^{3}}{2 \mu^{3}}\left(|y|^{2}\left(2+x_{4}^{2}\right)+y_{4}^{2}\right)+\mathcal{O}\left(\varepsilon^{2}\right), \quad \mathcal{W}=\varepsilon \frac{L^{3}}{2 \mu^{3}}|y|\left(4-x_{4}\right) y_{4} .
$$

Due to the regularization and the application of the invariants $a, b$ together with the pair $E / P_{E}$ we can handle the analysis of all kind of bounded motions through symplectic reduction.

\section{NORMALIZING AN N-DIMENSIONAL PERTURBED KEPLERIAN SYSTEM}

\subsection{Reduced Space of the $n$-Dimensional Kepler Problem}

The previous section can be generalized to any dimension without resorting to action-angle coordinates. Specifically one starts with the Kepler Hamiltonian with dimension $n \geq 2$ to which one attaches a smooth perturbation in rectangular coordinates and then applies Moser mapping (2.2). A generalization of the mapping (4.8) would yield the regularized perturbed Hamiltonian that would be written in terms of the corresponding invariants and the pair of angle-action coordinates given by $E / P_{E}$ with $P_{E}=|y|$, similarly to the spatial case. A normalization process would eliminate $E$ up to a certain order.

It seems natural to construct the invariants of the $n$-dimensional Kepler problem that parameterize the reduced space. Besides the Keplerian invariants we have to provide the fundamental constraints that relate them. We emphasize that the reduced space is a symplectic manifold since the reduction process lies within the context of regular symplectic reduction by Meyer [31] and Marsden and Weinstein [30].

Theorem 1. In the phase space $T^{+} \mathcal{S}^{n}$ the Keplerian invariants associated to the $n$-dimensional regularized Kepler Hamiltonian given in (2.7) are taken as the components of the angular momentum tensor plus the components of a scaled Laplace-Runge-Lenz vector. It leads to $n(n+1) / 2$ invariants, which in terms of Moser coordinates, are the quadratic expressions:

$$
a_{i, j}=x_{i} y_{j}-x_{j} y_{i}, \quad 1 \leq i<j \leq n+1 .
$$


These invariants are related through the $\left(n^{2}-3 n+4\right) / 2$ fundamental constraints given by:

$$
\begin{aligned}
& a_{1,2} a_{3,4}=a_{1,3} a_{2,4}-a_{1,4} a_{2,3}, \\
& \ldots \\
& a_{1,2} a_{3, n+1}=a_{1,3} a_{2, n+1}-a_{1, n+1} a_{2,3}, \\
& a_{1,2} a_{4,5}=a_{1,4} a_{2,5}-a_{1,5} a_{2,4}, \\
& \ldots \\
& a_{1,2} a_{4, n+1}=a_{1,4} a_{2, n+1}-a_{1, n+1} a_{2,4}, \\
& \ldots \\
& a_{1,2} a_{n-1, n}=a_{1, n-1} a_{2, n}-a_{1, n} a_{2, n-1}, \\
& a_{1,2} a_{n-1, n+1}=a_{1, n-1} a_{2, n+1}-a_{1, n+1} a_{2, n-1}, \\
& a_{1,2} a_{n, n+1}=a_{1, n} a_{2, n+1}-a_{1, n+1} a_{2, n}, \\
& \sum_{1 \leq i<j \leq n+1} a_{i, j}^{2}=P_{E}^{2},
\end{aligned}
$$

where $\left|a_{i, j}\right| \leq P_{E}$.

The reduced space associated to (2.7) is a symplectic manifold of dimension $2(n-1)$ diffeomorphic to $G_{2, n+1}$ and it is parameterized by the $n(n+1) / 2$ invariants $a_{i, j}$ together with the constraints (5.2) where in the last restriction we make $P_{E}=L$ with $L=\mu / k$ a positive parameter.

Proof (of Theorem 1). The angular momentum tensor $q \wedge p$ is an integral of motion for the Hamiltonian function (2.7). It has $n(n-1)$ non-null entries from which we select $n(n-1) / 2$, that are the terms of the form $a_{i, j}=q_{i} p_{j}-q_{j} p_{i}$ with $1 \leq i<j \leq n$, see [23, 37, 50]. Applying (2.3) to $a_{i, j}$ we get that $q_{i} p_{j}-q_{j} p_{i}=x_{i} y_{j}-x_{j} y_{i}$.

The Laplace-Runge-Lenz vector is another integral of (2.7). It is explicitly defined by the $n$ dimensional vector

$$
A=\frac{1}{\mu}\left(|p|^{2} q-(q \cdot p) p\right)-\frac{q}{|q|},
$$

which is the generalization of (4.6) to any $n$. The application of (2.3) to $A$, restricting the calculations to the level set $\mathcal{H}_{\varepsilon}^{-1}\left(-k^{2} / 2\right)$ followed by the simplifications using the constraints involving $x$ and $y$, leads to

$$
A=\frac{k}{\mu}\left(x_{1} y_{n+1}-x_{n+1} y_{1}, x_{2} y_{n+1}-x_{n+1} y_{2}, \ldots, x_{n} y_{n+1}-x_{n+1} y_{n}\right) .
$$

We define the remaining $n$ invariants as

$$
A^{*}=\left(a_{1, n+1}, \ldots, a_{n, n+1}\right)=\frac{\mu}{k} A .
$$

Therefore we have obtained the $n(n+1)$ invariants given by $a_{i, j}$ with $1 \leq i<j \leq n+1$. They are independent functions [50]. Moreover they are global coordinates that parameterize the reduced space, describing all type of bounded motions. Notice that the Keplerian invariants in terms of the rectangular coordinates are given by the combinations $q_{i} p_{j}-q_{j} p_{i}$ together with the components of $A^{*}$ put in terms of the entries of $A$ in (5.3). 
Choosing six different invariants, namely $a_{i, j}, a_{k, l}, a_{i, k}, a_{j, l}, a_{i, l}, a_{j, k}$, it is easy to notice by inspection that $a_{i, j} a_{k, l}=a_{i, k} a_{j, l}-a_{i, l} a_{j, k}$. There are basically $(n+1) n(n-1)(n-2) / 24$ different constraints relating them where the subindices satisfy $1 \leq i<j<k<l \leq n+1$. We select those such that $i=1, j=2$ and $2<k<l \leq n+1$, thus we obtain $a_{1,2} a_{k, l}=a_{1, k} a_{2, l}-a_{1, l} a_{2, k}$ and there are $(n-1)(n-2) / 2$ of such constraints. Using the identities $|x|=1$ and $x \cdot y=0$, we get

$$
a_{1,2}^{2}+\cdots+a_{1, n+1}^{2}+a_{2,3}^{2}+\cdots+a_{2, n+1}^{2}+\cdots+a_{n-2, n+1}^{2}+a_{n-1, n+1}^{2}+a_{n, n+1}^{2}=|y|^{2} .
$$

Fixing a value for $|y|$ which is an integral of motion of $(2.7)$, say $|y|=P_{E}>0$ we conclude that the last formula of $(5.2)$ holds. We arrive at $n(n+1) / 2$ different invariants related through $\left(n^{2}-3 n+4\right) / 2$ constraints, which is the right number for a space of dimension $2(n-1)$. These constraints are independent functions in the reduced space because their differentials are linearly independent, in other words the rank of the matrix with dimensions $\left(n^{2}-3 n+4\right) / 2 \times n(n+1) / 2$ formed by the derivatives of the constraints with respect to the invariants is $\left(n^{2}-3 n+4\right) / 2$. From (5.5) we get $\left|a_{i, j}\right| \leq|y|$, therefore bounds $\left|a_{i, j}\right| \leq P_{E}$ are fulfilled.

Setting $P_{E}=\mu / k=L$, we consider that the geodesic flow on the $n$-sphere $\mathcal{S}^{n}$, i.e, $\mathcal{M}=T^{+} \mathcal{S}^{n}$ has associate Hamiltonian function, $\mathcal{H}_{0}: \mathcal{M} \rightarrow \mathbb{R}:(x, y) \mapsto|y|$ (i.e., $\mathcal{H}_{0}(x, y)$ is the length of the vector $y), \mathcal{N}=\{(x, y) \in \mathcal{M}:|y|=L\}$ is the $L$-sphere bundle and the reduced space is $G_{2, n+1}$, the Grassmannian manifold of oriented 2-planes in $\mathbb{R}^{n+1}$, see for instance [35] and also [3, 4, 51].

When $n=3$, the invariants $a, b$ are related to the $a_{i, j}$ of (5.1) through formulae (4.9). Specifically we get:

$$
\begin{gathered}
a_{1}=a_{1,4}+a_{2,3}, \quad a_{2}=-a_{1,3}+a_{2,4}, \quad a_{3}=a_{1,2}+a_{3,4}, \\
b_{1}=-a_{1,4}+a_{2,3}, \quad b_{2}=-a_{1,3}-a_{2,4}, \quad b_{3}=a_{1,2}-a_{3,4} .
\end{gathered}
$$

In the spatial case we prefer to handle the invariants $a$ and $b$ instead of $a_{i, j}$ in order to compare the results with those obtained in $[33,51]$.

\subsection{Normalization in the Invariants of $G_{2, n+1}$}

We start by generalizing the perturbed Keplerian Hamiltonians of Section 4 given by (4.1), (4.2) and (4.3) to any $n$. We fix an energy value, say $h=-k^{2} / 2<0$, working on the energy level $\mathcal{H}_{\varepsilon}=h$ and introduce the Hamiltonian

$$
\tilde{\mathcal{H}}_{\varepsilon}(q, p)=\frac{|q|}{k}\left(\mathcal{H}_{\varepsilon}(q, p)+\frac{k^{2}}{2}\right)+\frac{\mu}{k},
$$

to which we apply $\Phi_{\mathrm{M}}$ arriving at

$$
\mathcal{H}_{\varepsilon}^{*}(x, y)=|y|+\varepsilon \mathcal{P}^{*}(x, y ; \varepsilon),
$$

where this time the function $f$ such that $\mathcal{P}^{*}(x, y ; \varepsilon)=f(x, y) \mathcal{P}(x, y ; \varepsilon)$ becomes

$$
f(x, y)=\frac{1}{k^{2}} \sqrt{\sum_{i=1}^{n}\left(y_{i}+y_{n+1} x_{i}-y_{i} x_{n+1}\right)^{2}} .
$$

We present the normalization procedure of perturbed Hamiltonian systems in any dimension by means of the invariants of the Kepler problem and the action-angle pair $P_{E} / E$.

Theorem 2. The regularized Hamiltonian (5.6) is normalized with respect to the angle coordinate E after applying to it the map

$$
\begin{aligned}
& q_{i}=\frac{L}{\mu} \frac{1}{\left|A^{*}\right|}\left(a_{i, n+1}\left(P_{E} \cos E-\left|A^{*}\right|\right)-\left(C_{i} \cdot A^{*}\right) \sin E\right), \\
& p_{i}=\frac{\mu}{L} \frac{1}{\left|A^{*}\right|\left(\left|A^{*}\right| \cos E-P_{E}\right)}\left(\left(C_{i} \cdot A^{*}\right) \cos E+a_{i, n+1} P_{E} \sin E\right),
\end{aligned}
$$


$i=1, \ldots, n$. The vectors $C_{i}$ are defined as $\left(-a_{1, i}, \ldots,-a_{i-1, i}, 0, a_{i, i+1}, \ldots, a_{i, n}\right), i=1, \ldots, n$ while $A^{*}$ is given in (5.4).

The procedure is carried out by means of the Lie transformation method for Hamiltonians by normalizing the perturbation with respect to $E$ and taking into consideration that $\mathcal{H}^{*}=P_{E}$. The corresponding calculations of higher orders make use of the Poisson structure among the $a_{i, j}, E$ and $P_{E}$, which is given by

$$
\begin{aligned}
& \left\{a_{i, j}, a_{i, l}\right\}=a_{j, l}(j<l), \quad\left\{a_{i, j}, a_{i, l}\right\}=-a_{l, j}(l<j), \\
& \left\{a_{i, j}, a_{j, l}\right\}=-a_{i, l}, \\
& \left\{a_{i, j}, a_{k, i}\right\}=a_{k, j}, \\
& \left\{a_{i, j}, a_{k, j}\right\}=-a_{k, i}(k<i), \quad\left\{a_{i, j}, a_{k, j}\right\}=a_{i, k}(i<k), \\
& \left\{a_{i, j}, a_{k, l}\right\}=0, \text { for any other combination of } i, j, k, l, \\
& \left\{a_{i, j}, E\right\}=0, i, j=1, \ldots, n, \\
& \left\{a_{i, n+1}, E\right\}=-\frac{a_{i, n+1} P_{E}}{\left|A^{*}\right|^{2}}, i=1, \ldots, n, \\
& \left\{a_{i, j}, P_{E}\right\}=0, \quad\left\{E, P_{E}\right\}=1 .
\end{aligned}
$$

If the perturbation $\mathcal{P}^{*}(x, y ; \varepsilon)$ in $(5.7)$ is smooth on $T^{+} \mathcal{S}^{n}$ then the normalization is performed in the whole $T^{+} \mathcal{S}^{n}$.

Proof (of Theorem 2). We start by inverting the map (5.1), writing down Moser coordinates in terms of the invariants $a_{i, j}$ and $E, P_{E}$. This can be achieved after introducing explicitly $E$ and $P_{E}$. Thus we set $P_{E}=|y|$ and define $E$ by putting $\cos E$ and $\sin E$ in terms of $x_{n+1}, y_{n+1}$. Analogously to what we got in (4.8) for $n=3$ we set $x_{n+1}=\left(\left|A^{*}\right| / P_{E}\right) \cos E$ and $y_{n+1}=-\left|A^{*}\right| \sin E$. Next we make $x_{k}=O_{k} \cos E+Q_{k} \sin E$ and $y_{k}=R_{k} \cos E+S_{k} \sin E$ for $k=1, \ldots, n$ where $O_{k}, Q_{k}$, $R_{k}$ and $S_{k}$ are undetermined functions of $a_{i, j}$ and $P_{E}$. We replace the expressions of $x_{k}, y_{k}$, $x_{n+1}$ and $y_{n+1}$ in the equations $x_{k} y_{n+1}-x_{n+1} y_{k}=a_{k, n+1}$ and solve for the unknowns, obtaining $O_{k}=-S_{k} / P_{E}, Q_{k}=-a_{k, n+1} /\left|A^{*}\right|$ and $R_{k}=-a_{k, n+1} P_{E} /\left|A^{*}\right|$. In order to determine the functions $S_{k}, k=1, \ldots, n$, we make $x_{1} y_{k}-x_{k} y_{1}=a_{1, k}$ where $O_{k}, Q_{k}$ and $R_{k}$ are written in terms of $S_{k}$ and $a_{i, j}$. We get $S_{k}=\left(S_{1} a_{k, n+1}-\left|A^{*}\right| a_{1, k}\right) / a_{1, n+1}$ for $k=2, \ldots, n$. To obtain $S_{1}$ we impose $|x|=1$ with the expressions of $x_{k}, y_{k}$ put in terms of $a_{i, j}, S_{1}$ and $\cos E, \sin E$ (firstly $O_{k}$ is expressed in terms of $\left.S_{1}\right)$ and arrive at $S_{1}=\left(C_{1} \cdot A^{*}\right) /\left|A^{*}\right|$, from which we deduce that $S_{k}=\left(C_{k} \cdot A^{*}\right) /\left|A^{*}\right|$. Finally, the map relating Moser coordinates with the Keplerian invariants is written as:

$$
\begin{aligned}
x_{i} & =-\frac{1}{\left|A^{*}\right|}\left(\frac{\left(C_{i} \cdot A^{*}\right)}{P_{E}} \cos E+a_{i, n+1} \sin E\right), \quad i=1 \ldots, n, \\
y_{i} & =\frac{1}{\left|A^{*}\right|}\left(-a_{i, n+1} P_{E} \cos E+\left(C_{i} \cdot A^{*}\right) \sin E\right), \quad i=1 \ldots, n, \\
x_{n+1} & =\frac{\left|A^{*}\right|}{P_{E}} \cos E \\
y_{n+1} & =-\left|A^{*}\right| \sin E .
\end{aligned}
$$

Formulae (5.10) make sense provided $\left|A^{*}\right| \neq 0$. Besides, one has $A^{*}=0$ if and only if $\left|A^{*}\right|=0$ and it corresponds to the case

$$
x_{n+1}=y_{n+1}=0, \quad x_{1}^{2}+\cdots+x_{n}^{2}=1, \quad x_{1} y_{1}+\cdots+x_{n} y_{n}=0, \quad y_{1}^{2}+\cdots+y_{n}^{2}=P_{E}^{2} .
$$


This case is the generalization of circular motions. In principle one can apply the mapping (5.10) and check if the formulae obtained after simplifying are singular for $\left|A^{*}\right|=0$. If this happens one should return to Moser coordinates. (5.8).

The composition of Moser map (2.2) with (5.10), after some simplifications, leads to the map

The transformation (5.8) is not well defined when $\left|A^{*}\right|=0$ and when $\left|A^{*}\right| \cos E=P_{E}$. This latter situation corresponds to the collision state because it implies $x_{n+1}=1$ and $x_{1}=\ldots=x_{n}=0$, thus $x=$ np. Besides, since $\left|A^{*}\right| \leq P_{E}$ then $\cos E=1, \sin E=0, a_{i, j}=0$ for $1 \leq i<j \leq n$ and $C_{i}=0$. It is emphasized that if the perturbation is linear in $p_{i}$ and polynomial in $q_{i}$, then $\left|A^{*}\right| \cos E-P_{E}=-(\mu / L)|q|$ and the multiplication of $\mathcal{P}$ by $|q| / r$ makes that $\mathcal{P}^{*}$ is a finite trigonometric Fourier series in $E$.

In terms of the invariants $a_{i, j}$, "circular" motions satisfy $a_{i, n+1}=0$, for $i=1, \ldots, n$, therefore we obtain $n(n-1) / 2$ different invariants. Moreover, the $\left(n^{2}-3 n+4\right) / 2$ restrictions of $(5.2)$ get reduced to $\left(n^{2}-5 n+8\right) / 2$ fundamental constraints, hence the dimension of the set containing this type of motions is $2(n-2)$, which for $n=2$ represents a point in the reduced space and when $n=3$ it is a two-dimensional set diffeomorphic to $\mathcal{S}^{2}$, that is, the set $\mathcal{C}$ of Section 4.2.

Rectilinear motions $(q \wedge p=0)$ and the collision state $(q=0)$ satisfy $a_{i, j}=0$ for $1 \leq i<j \leq n$. Moreover $a_{i, n+1}=x_{i} y_{n+1}-x_{n+1} y_{i}=-y_{i}, i=1, \ldots, n$ and the set of restrictions (5.2) gets reduced to only one, namely, $\sum_{i=1}^{n} a_{i, n+1}^{2}=P_{E}^{2}$, thus this type of motions are represented in the reduced space by an $(n-1)$-dimensional set which is diffeomorphic to $\mathcal{S}^{n-1}$.

Plugging (5.10) in (5.7), equivalently (5.8) in (5.6), our regularized perturbed Hamiltonian $\mathcal{P}^{*}\left(a_{i, j}, E, P_{E} ; \mu, L, \varepsilon\right)$ is ready in order to perform the normalization with respect to the angle coordinate $E$. We stress that by virtue of the transformation (5.8), $L$ is a parameter for (5.10) and the normalization is performed as an average with respect to $E$, where one needs to take into account that $P_{E}$ is the conjugate momentum to $E$. One needs to introduce the Poisson brackets among the $a_{i, j}$ and $E$. The relations (5.9) are obtained straightforwardly from the Poisson structure of Moser coordinates (4.13), which is trivially extended to any $n$. The Poisson brackets $\left\{a_{i, j}, E\right\}$ are determined after putting $E$ in terms of $x$ and $y$ by means of

$$
\cos E=\frac{|y| x_{n+1}}{\sqrt{|y|^{2} x_{n+1}^{2}+y_{n+1}^{2}}}, \quad \sin E=-\frac{y_{n+1}}{\sqrt{|y|^{2} x_{n+1}^{2}+y_{n+1}^{2}}},
$$

which is deduced from the last two formulae of (5.10).

Once the higher-order terms are discarded, $P_{E}$ becomes a constant of motion and the transformed Hamiltonian is an autonomous function of the invariants $a_{i, j}$ independent of $E$, thus all type of bounded motions can be analyzed in the reduced space. In fact, whether the perturbation in (5.7) is smooth on $T^{+} \mathcal{S}^{n}$ the normalization can be achieved in the whole space in Moser coordinates.

As a final step one restricts the normalized Hamiltonian to the adequate energy level by setting $P_{E}=L$. This Hamiltonian is defined in the reduced space, that is the symplectic manifold $G_{2, n+1}$, by means of the global coordinates $a_{i, j}$ and the constraints of (5.2).

From the point of view of the applications one has to calculate the Gröbner basis formed by the invariants $a_{i, j}$ written in terms of $q, p$ (or in terms of $x$ and $y$ if one decides to work with Moser coordinates) as well as all the $(n+1) n(n-1)(n-2) / 24$ constraints among the invariants plus the one of formula (5.5). Then, given a function (usually a polynomial) in terms of rectangular (or Moser) coordinates one applies the multivariate division algorithm with respect to the Gröbner basis, and the remainder of it yields this function in terms of the invariants alone. This procedure has been applied successfully in $[41-43,46]$ in the setting of the three-body problem. Similarly, the generating functions appearing when pushing the normalization to higher orders can be simplified in the same way, taking into account that they are finite Fourier series in $E$ whose coefficients are functions of the invariants $a_{i, j}$. 
Remark 4. As we did in Remark 3, we compare our procedure with the approach of van der Meer and Cushman [50]. One gets equivalent formulae with both methods. By means of (5.1) we pass from the normalized Hamiltonian and its generating function to Moser coordinates. The normalization in Moser coordinates usually leads to shorter calculations. Moreover, as long as $n$ increases the number of the invariants $a_{i, j}$ and the related constraints grows quickly while dealing with $x, y$ one always has $2 n+2$ coordinates with three restrictions and may avoid the indetermination occurring for $\left|A^{*}\right|=0$. As a consequence, a wise idea seems to be to perform the whole process in Moser coordinates, applying van der Meer and Cushman's approach and apply (5.10) to get the normalized Hamiltonian as a function independent of $E$, that is, in the reduced space. We also set $P_{E}=L$ to restrict the Hamiltonian to the right energy level. Notice that working with Moser coordinates involves the use of the Poisson brackets (4.13). Alternatively, one can proceed in terms of $x, y$ for the intermediate computations and at the time of getting the average and the generating function, apply (5.10) and normalize with respect to $E$. After obtaining the normal form Hamiltonian and the generating function in terms of the invariants one returns to $x$ and $y$ by means of (5.1), simplifying the resulting expressions through the application of Gröbner bases.

\section{APPLICATION TO THE LUNAR CASE OF THE RESTRICTED PROBLEM}

\subsection{The Problem, Regularization and Averaging}

The Hamiltonian of the spatial circular restricted three-body problem is given in a rotating frame by the function

$$
\mathcal{H}=\frac{1}{2}\left(p_{1}^{2}+p_{2}^{2}+p_{3}^{2}\right)-\left(q_{1} p_{2}-q_{2} p_{1}\right)-\frac{\bar{\mu}}{\sqrt{\left(q_{1}-1+\bar{\mu}\right)^{2}+q_{2}^{2}+q_{3}^{2}}}-\frac{1-\bar{\mu}}{\sqrt{\left(q_{1}+\bar{\mu}\right)^{2}+q_{2}^{2}+q_{3}^{2}}},
$$

where $\bar{\mu}$ represents the mass parameter given by $\bar{\mu}=m_{1} /\left(m_{1}+m_{2}\right)$ such that $m_{1}$ and $m_{2}$ refer to the masses of the primaries and it is assumed that $m_{1} \geq m_{2}$. See [32] and also [10, 51].

We change coordinates in order to bring $\mathcal{H}$ into a suitable form. By doing so we restrict $\mathcal{H}$ to a particular regime where the infinitesimal particle is moving around one of the primaries, the so called lunar case of the circular spatial restricted three-body problem. The details can be seen in [25], see also [51]. The transformed Hamiltonian is the function

$$
\mathcal{H}_{\varepsilon}=\frac{1}{2}\left(p_{1}^{2}+p_{2}^{2}+p_{3}^{2}\right)-\frac{1}{\sqrt{q_{1}^{2}+q_{2}^{2}+q_{3}^{2}}}-\varepsilon^{3}\left(q_{1} p_{2}-q_{2} p_{1}\right)+\frac{1}{2} \varepsilon^{6} \bar{\mu}\left(-2 q_{1}^{2}+q_{2}^{2}+q_{3}^{2}\right)+\mathcal{O}\left(\varepsilon^{8}\right) .
$$

Our Hamiltonian is already written in the desired form, that is, as a perturbation of the spatial Kepler problem with mass parameter $\mu=1$ and our aim is to apply the procedure of the previous sections. We have performed the normalization with respect to the mean anomaly in [33] but in the context of Ligon-Schaaf regularization, thus the problem with the loss of analyticity for $\ell=0$ when $G=0$ (i.e., at the collision state) remains. Therefore we need to resolve this issue in order to conclude the existence of invariant 3-tori of rectilinear type, and eventually the vertical periodic solutions of [51].

We first proceed in mixed polar-nodal and Delaunay coordinates. Expressing $\mathcal{H}_{\varepsilon}$ in these two sets of variables, we get

$$
\begin{aligned}
\mathcal{H}_{\varepsilon}= & -\frac{1}{2 L^{2}}-\varepsilon^{3} N \\
& +\frac{1}{8} \varepsilon^{6} \bar{\mu} r^{2}\left(1-3 \bar{c}^{2}-3\left(1-\bar{c}^{2}\right) \cos (2 \vartheta)+6 \bar{c} \sin (2 \vartheta) \sin (2 \nu)\right. \\
& \left.\quad-3\left(1-\bar{c}^{2}+\left(1+\bar{c}^{2}\right) \cos (2 \vartheta)\right) \cos (2 \nu)\right)+\mathcal{O}\left(\varepsilon^{8}\right) .
\end{aligned}
$$

In [33] we normalized Hamiltonian $\mathcal{H}_{\varepsilon}$ with respect to the mean anomaly $\ell$ and reduced the resulting system after truncating terms of order $\mathcal{O}\left(\varepsilon^{8}\right)$. Here we apply Moser regularization described in Section 2 in order to make the approach valid even for rectilinear motions. The procedure is carried out by fixing a negative value of the energy, say $h$, so that we introduce $k>0$ with $h=-k^{2} / 2$. Besides we apply the transformation (3.3) expressing the Hamiltonian in 
Delaunay-like coordinates. The unperturbed term (the spatial Kepler Hamiltonian) is converted into $P_{E}$ whereas the perturbation, i.e., the terms factorized by $\varepsilon^{3}$ and higher powers are multiplied by $r / k=L r$. After some simplifications, the Hamiltonian reads as

$$
\begin{array}{rl}
\mathcal{H}_{\varepsilon}^{*}=P_{E}-\varepsilon^{3} L^{2} P_{E} & N(1-\tilde{e} \cos E) \\
-\frac{1}{16} \varepsilon^{6} \bar{\mu} L^{4} P_{E}^{3}(1-\tilde{e} \cos E)\left(\left(3 \bar{c}^{2}-1\right)\left(\tilde{e}^{2}-4 \tilde{e} \cos E+2\right)\right. \\
+6 \bar{s}^{2}(1-\tilde{e} \cos E)^{2} \cos (2 \nu) \\
+6 \sin (2 g)\left(2 \tilde{\eta}(\tilde{e}-\cos E)\left(\bar{s}^{2}-\left(\bar{s}^{2}-2\right) \cos (2 \nu)\right) \sin E\right. \\
+\bar{c} \tilde{e}(4 \cos E-3 \tilde{e}) \sin (2 \nu)) \\
+3 \cos (2 g)\left(\tilde{e}(3 \tilde{e}-4 \cos E)\left(\bar{s}^{2}-\left(\bar{s}^{2}-2\right) \cos (2 \nu)\right)\right. \\
+8 \bar{c} \tilde{\eta}(\tilde{e}-\cos E) \sin E \sin (2 \nu)) \\
+\cos (2 E)\left(\tilde{e}^{2}\left(2-3 \bar{s}^{2}\right)\right. \\
+3\left(\tilde{e}^{2}-2\right)\left(\cos (2 g)\left(\left(\bar{s}^{2}-2\right) \cos (2 \nu)-\bar{s}^{2}\right)\right. \\
+2 \bar{c} \sin (2 g) \sin (2 \nu))))+\mathcal{O}\left(\varepsilon^{8}\right) .
\end{array}
$$

Remark 5. Hamiltonian $\mathcal{H}_{\varepsilon}^{*}$ can be interpreted as the regularized Hamiltonian related to $\mathcal{H}$ in (6.1) after changing coordinates and introducing a small parameter. In fact, starting from $\mathcal{H}$, we could have defined the intermediate $\mathcal{H}^{*}$ as

$$
\mathcal{H}^{*}=\frac{|q|}{k}\left(\mathcal{H}+\frac{k^{2}}{2}\right)+\frac{1}{k} .
$$

Then, one could have applied Moser map (2.2) to $\mathcal{H}^{*}$ and introduce the small parameter $\varepsilon$ together with the adequate transformations in terms of $x$ and $y$ in order to define the lunar problem. Then, after passing from rectangular to polar-nodal and Delaunay-like coordinates we would have obtained the same Hamiltonian $\mathcal{H}_{\varepsilon}^{*}$ of $(6.3)$.

Hamiltonian $\mathcal{H}_{\varepsilon}^{*}$ may be viewed as an analytic function of $\tilde{e}, \tilde{\eta}, \cos \nu, \sin \nu, G, N, P_{E}, \cos E$ and $\sin E$. Indeed it is $2 \pi$-periodic with respect to the eccentric anomaly $E$. It also depends on the parameters $L$ and $\bar{\mu}$. Alternatively one can start with (6.2), apply first the mapping (2.2) and then (3.9). After simplifying we arrive at the same Hamiltonian function $\mathcal{H}_{\varepsilon}^{*}$.

Normalizing the Hamiltonian with respect to $E$ applying Lie transformations where the unperturbed term is $P_{E}$, see for instance $[13,32]$, we end up with

$$
\begin{array}{rl}
\mathcal{H}_{\varepsilon}^{*}=P_{E}-\varepsilon^{3} L^{2} P_{E} & N \\
+\frac{1}{16} \varepsilon^{6} L^{4} P_{E}^{3}( & -8 \bar{c}^{2} \tilde{\eta}^{2}+30 \bar{\mu} \bar{c} \tilde{e}^{2} \sin (2 g) \sin (2 \nu) \\
& +\bar{\mu}\left(2+3 \tilde{e}^{2}\right)\left(1-3 \bar{c}^{2}-3\left(1-\bar{c}^{2}\right) \cos (2 \nu)\right) \\
& \left.-15 \bar{\mu} \tilde{e}^{2} \cos (2 g)\left(1-\bar{c}^{2}+\left(1+\bar{c}^{2}\right) \cos (2 \nu)\right)\right)+O\left(\varepsilon^{8}\right) .
\end{array}
$$

To complete the normalization process one has to set $P_{E}=L$ in order to adjust the right energy level. The resulting Hamiltonian keeps the same name.

If we drop the unperturbed terms in the expression of $\mathcal{H}_{\varepsilon}$ of Eq. (12) in reference [33] and in $\mathcal{H}_{\varepsilon}^{*}$, set $P_{E}=L$, call the transformed Hamiltonians with the same names and compare them, we end up with the identity:

$$
\mathcal{H}_{\varepsilon}=m\left(\mathcal{H}_{\varepsilon}^{*}+\frac{1}{2} \varepsilon^{6} L^{5} N^{2}\right)
$$


with $m=1 / L^{3}$, at least up to terms in $\mathcal{O}\left(\varepsilon^{8}\right)$. This Hamiltonian in normal form is well defined for rectilinear motions, but one should replace the angles $g$ and $\nu$ by other angles which make sense for this type of motions, see for instance [49]. We shall see below that the extra term of $\mathcal{H}_{\varepsilon}^{*}$ compared to $\mathcal{H}_{\varepsilon}$ does not essentially modify the dynamics of the reduced system.

Alternatively we may work with the Keplerian invariants $a$ and $b$. After regularizing, but before normalization, we make the replacement of $q$ and $p$ in (6.2) in terms of $E, P_{E}, a, b$ and the parameter $L$. The resulting Hamiltonian is a function of the invariants and $P_{E}$ whose coefficients are finite Fourier series in $E$ that we normalize with respect to $E$ up to terms of order $\mathcal{O}\left(\varepsilon^{6}\right)$, getting

$$
\begin{aligned}
\mathcal{H}_{\varepsilon}^{*}= & P_{E}-\frac{1}{2} \varepsilon^{3} L^{2} P_{E}\left(a_{3}+b_{3}\right) \\
& -\frac{1}{8} \varepsilon^{6} L^{4} P_{E}\left(\left(a_{3}+b_{3}\right)^{2}+2 \bar{\mu}\left(3\left(a_{1}-b_{1}\right)^{2}+3 a_{2} b_{2}+3 a_{3} b_{3}-2 P_{E}^{2}\right)\right)+O\left(\varepsilon^{8}\right) .
\end{aligned}
$$

If we drop the unperturbed term in (6.6), set $P_{E}=L$ and scale the Hamiltonian dividing by $\varepsilon^{3}$, keep the same name for the Hamiltonian and compare it with Hamiltonian $\overline{\mathcal{H}}$ appearing in [51] (Formula (21)) or in [33] (Formula (15)), we get

$$
\overline{\mathcal{H}}=m\left(\mathcal{H}_{\varepsilon}^{*}+\frac{1}{8} \varepsilon^{6} L^{5}\left(a_{3}+b_{3}\right)^{2}\right) .
$$

We will see that the flow of the reduced system defined through $\overline{\mathcal{H}}$ and analyzed in [33, 51] agrees with the flow of the vector field related to the regularized reduced Hamiltonian $\mathcal{H}_{\varepsilon}^{*}$. Finally, notice that $\mathcal{H}_{\varepsilon}^{*}$ is analytic on the whole reduced space $\mathcal{S}^{2} \times \mathcal{S}^{2}$.

\subsection{Periodic Solutions of Rectilinear Type}

The vector field associated to (6.6) may be studied in order to extract some qualitative information of system (6.1), in particular to obtain periodic solutions, invariant tori and quasiperiodic solutions for the full problem. However the analysis provided by this vector field about the periodic solutions is the same as the one corresponding to the non-regularized version studied in $[33,51]$. The reason is that the existence of the periodic solutions is derived from the terms of order three in $\varepsilon$ and the Hamiltonian of $[33,51]$ agrees with $\mathcal{H}_{\varepsilon}^{*}$ up to $\varepsilon^{3}$.

In particular, in [51] we studied the flow associated to Hamiltonian $\mathcal{H}_{\varepsilon}$ on the reduced space $\mathcal{S}^{2} \times \mathcal{S}^{2}$ determining four relative equilibrium points of the type $(a, b)$, namely, $(0,0, \pm L, 0,0, \pm L)$ and $(0,0, \pm L, 0,0, \mp L)$. These equilibria remain the same when adding the additional terms in (6.7). The two first points correspond to circular coplanar motions whereas the other two correspond to rectilinear motions in the axis perpendicular to the equatorial plane. Applying Reeb's Theorem $[45,51]$ we concluded the existence of four families (one for each equilibrium) of periodic solutions for Hamiltonian (6.1) parameterized by the action $L$, whose periods are $T=2 \pi L^{3}+\mathcal{O}\left(\varepsilon^{3}\right)$. We also dealt with the stability character of these periodic solutions, proving that they were of elliptic type. Specifically, the periodic solutions nearly rectilinear generalize the solutions found by Belbruno [2] for small $\mu$, whereas the nearly circular equatorial solutions correspond to the well known circular periodic solutions of the planar restricted problem.

Far from the near-rectilinear solutions we do not need to use Moser regularization since the study made in [51] was fine. However for the rectilinear type of solutions the averaging process performed in [51] is not fully justified due to the loss of analyticity when $G=0$ at $\ell=0$ pointed out in Section 1. Thus we tackle this issue applying Moser regularization in the context of this paper.

Focusing on the points of rectilinear character, i.e., points with coordinates $(0,0, \pm L, 0,0, \mp L)$, we first notice that the averaging procedure performed is valid for this type of motions. In this case, due to the equivalence of the non-regularized and regularized reduced Hamiltonians established by means of (6.7), the analysis achieved in [51] is valid for the regularized system. Thus, as $\mathcal{H}_{\varepsilon}^{*}$ is a $2 \pi$ periodic function with respect to $E$, we conclude that the regularized Hamiltonian $\mathcal{H}^{*}$ corresponding to $\mathcal{H}$ in (6.1) has families of periodic solutions related to the points $(0,0, \pm L, 0,0, \mp L)$. Their periods are $T=2 \pi+\mathcal{O}\left(\varepsilon^{3}\right)$. 
Returning to Hamiltonian (6.2) the conclusion is that there are families of periodic solutions that are near-rectilinear but also near-perpendicular to the plane defined by the primaries whose periods are of the type $T=2 \pi L^{3}+\mathcal{O}\left(\varepsilon^{3}\right)$. These families are parameterized by $L$, or by the energy $h$. As studied in [51] the eccentricity of these solutions is $e=1-\frac{11025}{512} \varepsilon^{10} \bar{\mu}^{2}(1-\bar{\mu})^{2 / 3} L^{10}+\cdots$ while the length of the angular momentum vector is $G=\frac{105}{16} \varepsilon^{5} \bar{\mu}(1-\bar{\mu})^{1 / 3} L^{6}+\cdots$ and $N=$ $-\frac{33075}{512} \varepsilon^{13} \bar{\mu}(1-\bar{\mu})^{2 / 3} L^{14}+\cdots$. See more details in [51]. We stress that these values have not changed after adding the additional terms in (6.7).

\subsection{Invariant 3-Tori and Quasi-Periodic Solutions of Rectilinear Type}

To ensure the persistence of invariant 3-tori we can apply Han, Li and Yi's Theorem [21]. In particular we can achieve it without resorting to an isoenergetic version of the theorem, but performing the whole approach without restricting ourselves to a specific energy level. For this, we need to work with $P_{E}$ instead of $L$.

There are three families of KAM 3-tori related to near-rectilinear motions, see [33, 51]. Two of them correspond to the periodic solutions of the previous subsection, i.e., they are related to the equilibria $\left(0,0, \pm P_{E}, 0,0, \mp P_{E}\right)$, whereas the third one represents rectilinear motions in the equatorial plane. This last family is deduced from an equilibrium point on the twice-reduced space obtained after normalizing (6.4) over the argument of the node and reducing the resulting system accordingly. The essential analysis to achieve the persistence of these KAM tori is carried out in [33], in particular the rectilinear perpendicular solutions are studied in Section 4.3 of this paper while the rectilinear equatorial solutions are analysed in Section 5.3.3.

Due to the equivalence of the non-regularized and regularized reduced flows the normal form calculations and the analysis carried out in $[33,51]$ are adapted easily. One can also deal with a second normal form computation in order to normalize Hamiltonian (6.4) with respect to $\nu$ using the invariants $a$ and $b$ but starting with Hamiltonian (6.6), similarly to the strategy developed in reference [11].

\subsubsection{Persistence of the 3-tori of near-rectilinear perpendicular type}

Following $[33,51]$ we introduce two actions, namely $J_{1}$ and $J_{2}$, and write down the corresponding Hamiltonian system in a suitable form, beginning from Hamiltonian (6.6), we arrange it properly eliminating by averaging the dependence of the angles conjugate to $J_{i}$, i.e. $\psi_{i}$, and apply to it Han, $\mathrm{Li}$ and Yi's Theorem to conclude the persistence of invariant tori for the regularized Hamiltonian $\mathcal{H}^{*}$ using $J_{1}, J_{2}$ and $P_{E}$ for the third action. A similar process is applied to the family of near-rectilinear equatorial tori reconstructed from the second reduction.

Concretely for the equilibria $\left(0,0, \pm P_{E}, 0,0, \mp P_{E}\right)$ we proceed as follows. Firstly we move the origin to the relative equilibria

$$
a_{1}=\bar{a}_{1}, \quad a_{2}=\bar{a}_{2}, \quad a_{3}=\bar{a}_{3} \pm P_{E}, \quad b_{1}=\bar{b}_{1}, \quad b_{2}=\bar{b}_{2}, \quad b_{3}=\bar{b}_{3} \mp P_{E},
$$

and define the local symplectic coordinates

$$
Q_{1}=\frac{\bar{a}_{2}}{\sqrt{2 P_{E} \pm \bar{a}_{3}}}, \quad Q_{2}=\frac{\bar{b}_{2}}{\sqrt{2 P_{E} \mp \bar{b}_{3}}}, \quad P_{1}=\mp \frac{\bar{a}_{1}}{\sqrt{2 P_{E} \pm \bar{a}_{3}}}, \quad P_{2}= \pm \frac{\bar{b}_{1}}{\sqrt{2 P_{E} \mp \bar{b}_{3}}} .
$$

Now, the idea is to introduce a pair of action-angle variables $J_{j} / \psi_{j}$ from $Q, P$ and remove the dependence of the Hamiltonian on the angles $\psi_{1}, \psi_{2}$. The process is lengthy but customary and is described in $[33,51]$. After performing some manipulations the following normal form is obtained:

$$
\begin{aligned}
\mathcal{H}_{\varepsilon}^{*}= & P_{E}+\frac{1}{4} \varepsilon^{6} L^{2} P_{E}\left(5 \bar{\mu} L^{2} P_{E}^{2}+4\left(J_{1}-J_{2}\right)\right)+\frac{3 \sqrt{15}}{4} \varepsilon^{9} \bar{\mu} L^{4} P_{E}^{2}\left(J_{1}+J_{2}\right) \\
& +\frac{1}{32} \varepsilon^{12}\left(-16 L^{4} P_{E}\left(J_{1}-J_{2}\right)^{2}+48 \bar{\mu} L^{4} P_{E}\left(J_{1}+J_{2}\right)^{2}+45 \bar{\mu}^{2} L^{6} P_{E}^{3}\left(J_{1}-J_{2}\right)\right) \\
& +\mathcal{O}\left(\varepsilon^{15}\right) .
\end{aligned}
$$


The details on the introduction of the action-angle coordinates and how to obtain (6.9) are analogous to those given in [33]. Using the notation of [51] we take $a=3, m_{1}=6, m_{2}=9$ and $m_{3}=12, n_{0}=1, n_{1}=n_{2}=n_{3}=3, I^{n_{0}}=\left(P_{E}\right), I^{n_{1}}=I^{n_{2}}=I^{n_{3}}=\left(P_{E}, J_{1}, J_{2}\right), \bar{I}^{n_{0}}=\left(P_{E}\right)$, $\bar{I}^{n_{1}}=\bar{I}^{n_{2}}=\bar{I}^{n_{3}}=\left(J_{1}, J_{2}\right)$. Besides, $h_{0}$ is $P_{E}$ while $h_{1}, h_{2}$ and $h_{3}$ are the terms in (6.9) of order $\varepsilon^{6}$, $\varepsilon^{9}$ and $\varepsilon^{12}$, respectively. The vector of frequencies is given by

$$
\Omega(I)=\left(\frac{\partial h_{0}}{\partial P_{E}}, \frac{\partial h_{1}}{\partial J_{1}}, \frac{\partial h_{1}}{\partial J_{2}}, \frac{\partial h_{2}}{\partial J_{1}}, \frac{\partial h_{2}}{\partial J_{2}}, \frac{\partial h_{3}}{\partial J_{1}}, \frac{\partial h_{3}}{\partial J_{2}}\right)
$$

and the $4 \times 6$-matrix with rows $\Omega(I), \partial \Omega(I) / \partial P_{E}, \partial \Omega(I) / \partial J_{1}$ and $\partial \Omega(I) / \partial J_{2}$ has rank four, thus Hamiltonian (6.9) satisfies the hypotheses of Han, Li, Yi's Theorem. Thence, one concludes with the persistence of KAM 3-tori of rectilinear perpendicular type related to the vector field associated with $\mathcal{H}_{\varepsilon}^{*}$. Setting $P_{E}=L$ and returning to the coordinates prior to regularization one concludes the persistence of KAM 3-tori of rectilinear perpendicular type related to the vector field associated with $\mathcal{H}$ in (6.2). These invariant tori form a majority in the sense that the measure of the complement of their union is of order $O\left(\varepsilon^{12}\right)$.

\subsubsection{Persistence of the 3-tori of near-rectilinear equatorial type}

We follow the construction made in Section 5.3.3 of [33]. As a first step Hamiltonian (6.4) is averaged with respect to $\nu$. Then one applies singular reduction theory and puts the averaged Hamiltonian in terms of the global coordinates $\tau=\left(\tau_{1}, \tau_{2}, \tau_{3}\right)$ that define the twice-reduced space that we call $\mathcal{T}_{P_{E}, N}$. The equilibrium point of interest for the reduced Hamiltonian in the reduced space has coordinates $\tau=\left(0,0, N^{2}-P_{E}^{2}\right)$ and the invariant 3 -tori that are nearly rectilinear arise when $N \approx 0$.

In order to reconstruct this kind of tori we need a set of action-angle coordinates. Two candidates are $P_{E}$ and $N$ and the third one, that we name $J$, is built in the normal form process. The coordinates $P_{E}, N$ and $E$ behave properly for near-rectilinear motions and although $\nu$ is not well defined one can use the angle $\phi_{a}$, the longitude of aphelion, which is conjugate to $N$, see [49].

Specifically the construction of $J$ and its conjugate angle $\varphi$ is based on the form of the reduced Hamiltonian derived from Eq. (27) in [33] in the twice-reduced space. A previous step to get $J$ and $\varphi$ is to introduce a rectangular pair of local symplectic coordinates, $Q, P$, in terms of the invariants of the twice-reduced space $\mathcal{T}_{P_{E}, N}$. Concretely we set

$$
\begin{gathered}
\tau_{1}=P, \quad \tau_{2}=\sqrt{\left(P_{E}^{2}-(N+P)^{2}\right)\left(P_{E}^{2}-(N-P)^{2}\right)} \sin (2 Q), \\
\tau_{3}=-\sqrt{\left(P_{E}^{2}-(N+P)^{2}\right)\left(P_{E}^{2}-(N-P)^{2}\right)} \cos (2 Q)
\end{gathered}
$$

and $Q, P$ are adequate coordinates in a neighborhood of the point $\left(0,0, N^{2}-P_{E}^{2}\right)$.

The corresponding normal form Hamiltonian is

$$
\begin{aligned}
\mathcal{H}_{\varepsilon}^{*}= & P_{E}-\varepsilon^{3} L^{2} N P_{E}-\frac{1}{8} \varepsilon^{6} \bar{\mu} L^{4} P_{E}\left(5 \bar{\mu} P_{E}^{2}+(4-3 \bar{\mu}) N^{2}\right) \\
& +\frac{3 \sqrt{2}}{4} \varepsilon^{7} \bar{\mu} L^{4} P_{E} \sqrt{5 P_{E}^{2}-3 N^{2}} J+\mathcal{O}\left(\varepsilon^{8}\right) .
\end{aligned}
$$

The details on how to introduce $J$ and $\varphi$ in terms of $Q, P$ and get the normal form (6.11) appear in [33] (see in particular Section 5.3.3 and Eq. (38)). In the process of getting the normal form we apply the stretching $(\bar{Q}, \bar{P})=\varepsilon^{1 / 2}(Q, P)$ of $[33]$.

This time $a=3, m_{1}=3, m_{2}=6, m_{3}=7, n_{0}=1, n_{1}=n_{2}=2, n_{3}=3, I^{n_{0}}=\left(P_{E}\right), I^{n_{1}}=I^{n_{2}}=$ $\left(P_{E}, N\right), I^{n_{3}}=\left(P_{E}, N, J\right), \bar{I}^{n_{0}}=\left(P_{E}\right), \bar{I}^{n_{1}}=\bar{I}^{n_{2}}=(N), \bar{I}^{n_{2}}=(J), h_{0}=P_{E}$ and $h_{1}, h_{2}$ and $h_{3}$ correspond respectively to the terms in (6.11) of orders 3,6 and 7 in $\varepsilon$. The rank of the matrix formed by the frequency vector

$$
\Omega(I)=\left(\frac{\partial h_{0}}{\partial P_{E}}, \frac{\partial h_{1}}{\partial N}, \frac{\partial h_{2}}{\partial N}, \frac{\partial h_{3}}{\partial J}\right),
$$

REGULAR AND CHAOTIC DYNAMICS Vol. 00 No. $0 \quad 0000$ 
and its derivatives with respect to $P_{E}, N$ and $J$ is three. Thus, as it is proved in [33], Hamiltonian (6.11) satisfies Han, Li, Yi's Theorem hypotheses as before, establishing the persistence of KAM 3 -tori of rectilinear equatorial type related to $\mathcal{H}_{\varepsilon}^{*}$. Setting $P_{E}=L$ and going back to the nonregularized coordinates we get the persistence of KAM 3-tori of rectilinear equatorial type related to $\mathcal{H}$ given in (6.2). The measure of the excluded tori is of order $O\left(\varepsilon^{10}\right)$.

Remark 6. The solutions obtained in Sections 6.3.1 and 6.3.2 are quasi-periodic only in the regularized time $s$ but not in $t$.

\subsection{Checking the Transversality of the Manifolds}

The final step in the discussion of the periodic and quasi-periodic solutions in the previous subsections is to show that a set of positive measure of invariant tori intersect transversely the collision set in submanifolds of codimension at least two. The reason is that among those invariant tori found for the regularized system, the ones that could meet the collision set only form a set of relative measure of certain order of the small parameter $\varepsilon$, as it is studied in [29]. Hence our aim is to prove that most of the trajectories on the tori determined in the previous subsection pass arbitrarily close to the collision set but without intersection. We also apply this technique in [43].

\subsubsection{Transversality condition for the invariant tori of rectilinear perpendicular type}

We start by studying the transversality condition in the manifold $\mathcal{S}^{2} \times \mathcal{S}^{2}$ corresponding to $\left(0,0, \pm P_{E}, 0,0, \mp P_{E}\right)$. First of all, we observe that the invariant 3 -tori are projected into invariant 2 -tori with angles $\psi_{1}$ and $\psi_{2}$ and main frequencies given by the derivatives of $\mathcal{H}_{\varepsilon}^{*}$ with respect to $J_{1}$ and $J_{2}$ in (6.9).

It is not difficult to prove that near the points $\left(0,0, \pm P_{E}, 0,0, \mp P_{E}\right)$, the rectilinear motions expressed in terms of the coordinates $Q_{i}$ and $P_{i}$ introduced in (6.8), satisfy the conditions $Q_{1}=-Q_{2}$ and $P_{1}=P_{2}$. Applying the successive canonical changes required to get (6.9), up to terms of order three in $\varepsilon$, these conditions in the transformed coordinates become

$$
J_{1}=J_{2}+\frac{3 \sqrt{15}}{2} \varepsilon^{3} \bar{\mu} L^{2} P_{E} J_{2} \sin \left(2 \psi_{1}\right)+O\left(\varepsilon^{6}\right), \quad \psi_{1}+\psi_{2}=\frac{3 \pi}{2}+\frac{3 \sqrt{15}}{4} \varepsilon^{3} \bar{\mu} L^{2} P_{E} \cos \left(2 \psi_{1}\right)+O\left(\varepsilon^{6}\right) .
$$

We stop at order three because it is going to be enough for our purposes.

The collision set for the whole Hamiltonian system can be determined easily in the action/angle coordinates $\left(E, \psi_{1}, \psi_{2}, P_{E}, J_{1}, J_{2}\right)$. It is named $\mathcal{C}$ ol and is defined through

$$
\begin{aligned}
E & =O\left(\varepsilon^{6}\right), \\
\psi_{2} & =\frac{3 \pi}{2}-\psi_{1}+\frac{3 \sqrt{15}}{4} \varepsilon^{3} \bar{\mu} L^{2} P_{E} \cos \left(2 \psi_{1}\right)+O\left(\varepsilon^{6}\right), \\
J_{1} & =J_{2}+\frac{3 \sqrt{15}}{2} \varepsilon^{3} \bar{\mu} L^{2} P_{E} J_{2} \sin \left(2 \psi_{1}\right)+O\left(\varepsilon^{6}\right),
\end{aligned}
$$

while no condition is imposed to the rest of coordinates. We remark that $E$ has to be transformed while performing the changes of coordinates to get the normal form but its main significant variation occurs at order $\varepsilon^{6}$. The intersection of the set (6.12) with an invariant 3-torus obtained from (6.9), namely $\left(P_{E}, J_{1}, J_{2}\right)=\left(c_{1}, c_{2}, c_{3}\right)+\mathcal{O}\left(\varepsilon^{15}\right)$ with fixed $c_{i}>0$ are the points satisfying

$$
\begin{aligned}
& \left(E, \psi_{1}, \psi_{2}, P_{E}, J_{1}, J_{2}\right)= \\
& \quad\left(0, \psi_{1}, \frac{3 \pi}{2}-\psi_{1}+\frac{3 \sqrt{15}}{4} \varepsilon^{3} \bar{\mu} c_{1} L^{2} \cos \left(2 \psi_{1}\right), c_{1}, c_{3}+\frac{3 \sqrt{15}}{2} \varepsilon^{3} \bar{\mu} c_{1} c_{3} L^{2} \sin \left(2 \psi_{1}\right), c_{3}\right)+\mathcal{O}\left(\varepsilon^{6}\right),
\end{aligned}
$$

and the additional relation:

$$
c_{2}=c_{3}+\frac{3 \sqrt{15}}{2} \varepsilon^{3} \bar{\mu} c_{1} c_{3} L^{2} \sin \left(2 \psi_{1}\right)+\mathcal{O}\left(\varepsilon^{6}\right) .
$$


Assuming that $c_{2}$ is chosen as $c_{2}=c_{3}+\varepsilon^{3} c_{3}^{*}=c_{3}+\mathcal{O}\left(\varepsilon^{3}\right)$, the intersection of the collision manifold with the single 3 -torus are the four points obtained after solving (6.13) for $\psi_{1}$, say $\psi_{1}^{*, i}$ with $i \in\{1,2,3,4\}$, therefore it is of codimension 3 in the fixed torus.

The next step consists in calculating the normal vector spaces to the 3 -torus and to the set $\mathcal{C} o l$ at the intersection points and check that the intersection is transversal. It suffices to show that both vectors are not parallel, using the theory of intersection of manifolds [15]. We need to compute the Jacobians of the constraints that define both sets. Arranging these variables as before, i.e., putting $E, \psi_{1}, \psi_{2}, P_{E}, J_{1}, J_{2}$, the rows of the Jacobian matrices contain the partial derivatives of the corresponding constraints with respect to the six coordinates in the order we have established. For the 3 -torus at the intersection points we get

$$
J_{T}=\left(\begin{array}{cccccc}
0 & 0 & 0 & 1 & 0 & 0 \\
0 & 0 & 0 & 0 & 1 & 0 \\
0 & 0 & 0 & 0 & 0 & 1
\end{array}\right)+\mathcal{O}\left(\varepsilon^{6}\right)
$$

For the collision set we obtain

$$
J_{\mathcal{C} o l}=\left(\begin{array}{cccccc}
1 & 0 & 0 & 0 & 0 & 0 \\
0 & 1+\varepsilon^{3} k_{1} & 1 & 0 & 0 & 0 \\
0 & \varepsilon^{3} k_{2} & 0 & 0 & 1 & -1-\varepsilon^{3} k_{1}
\end{array}\right)+\mathcal{O}\left(\varepsilon^{6}\right)
$$

where $k_{1}=\frac{3 \sqrt{15}}{2} \bar{\mu} c_{1} L^{2} \sin \left(2 \psi_{1}^{*, i}\right)$ and $k_{2}=-3 \sqrt{15} \bar{\mu} c_{1} c_{3} L^{2} \cos \left(2 \psi_{1}^{*, i}\right)$. The normal vectors to the collision set and to the torus at the intersection points are given by the vector spaces spanned by the rows of the Jacobian matrices. They are, respectively,

$$
\begin{aligned}
\mathcal{N}_{T} & =\left(0,0,0, t_{4}, t_{5}, t_{6}\right)+\mathcal{O}\left(\varepsilon^{6}\right), \\
\mathcal{N}_{\mathcal{C}_{o l}} & =\left(s_{1}, s_{2}+\varepsilon^{3}\left(s_{2} k_{1}+s_{3} k_{2}\right), s_{2}, 0, s_{3},-s_{3}-\varepsilon^{3} s_{3} k_{1}\right)+\mathcal{O}\left(\varepsilon^{6}\right),
\end{aligned}
$$

where $s_{j}, t_{k}$ are arbitrary real numbers. Provided $k_{2} \neq 0$, it is readily deduced that the two vectors can be parallel only when $s_{i}=t_{i}=0$, thus the normal vectors intersect transversely. But $k_{2}=0$ is equivalent to $\psi_{1}^{*, 1}=\pi / 4, \psi_{1}^{*, 2}=3 \pi / 4, \psi_{1}^{*, 3}=5 \pi / 4, \psi_{1}^{*, 4}=7 \pi / 4$ and these situations can be avoided by selecting $c_{3}^{*}$ carefully so that when (6.13) is solved for $\psi_{1}$ we do not obtain the roots $\pi / 4,3 \pi / 4$, $5 \pi / 4,7 \pi / 4$.

The final step is the application of Lemma 7.4 in [52] which says that if $K$ is a submanifold of the $n$-torus $T_{n}$ having codimension at least two in $T_{n}$, then almost all the orbits of the linear flow defined by the angle coordinates on the torus do not intersect $K$. According to this lemma most of the trajectories on those tori intersecting $\mathcal{C}$ ol transversely do not intersect $\mathcal{C}$ ol. Moreover, since the flow is irrational on these invariant tori of $\mathcal{H}^{*}$, almost all the trajectories pass arbitrarily close to $\mathcal{C} o l$ without intersecting this set. A single (periodic or quasi-periodic) trajectory gives rise to a (periodic or quasi-periodic) solution of non-collision type of the system defined by Hamiltonian (6.2) that is close to the collision set $\left\{q_{1}=q_{2}=q_{3}=0\right\}$. Such motions form a set of positive measure where the massless particle passes arbitrarily close to one of the primaries without colliding with it but following a periodic or quasi-periodic motion.

\subsubsection{Transversality condition for the invariant tori of rectilinear equatorial type}

We observe that in the twice-reduced space $\mathcal{T}_{P_{E}, N}$ the rectilinear motions are given by $\left(\tau_{1}, 0, \tau_{3}\right)$ with $\tau_{3}=\tau_{1}^{2}-P_{E}^{2}$ and $N=0$, see also [33]. Translated to the coordinates $Q, P$ of (6.10) the transformed conditions where rectilinear motions occur become $Q=N=0$, thence in the angleaction pair we obtain $\varphi=\pi / 2$ and $N=0$.

We proceed analogously to the case of rectilinear perpendicular tori. An invariant 3-torus obtained from (6.11) is given by $\left(P_{E}, N, J\right)=\left(c_{1}, c_{2}, c_{3}\right)+\mathcal{O}\left(\varepsilon^{8}\right)$ where $c_{i}>0$. Then we have to 
determine the collision set in the transformed coordinates (also called normal form coordinates as they are the variables introduced to average over $\nu$ ) and the process is a bit laborious. We start by considering the corresponding set in the variables before the normal form transformation that has led to the averaging with respect to $\nu$. From the previous paragraph we deduce that the collision set is given by $E=0, \varphi=\pi / 2$ and $N=0$.

In order to get the collision set in the normal form coordinates $E, \phi_{a}, \varphi, N$ and $J$ we apply Lie transformations. The main difficulty in achieving it is that we calculated the generating function of the normal form transformation in terms of $g, \nu, G$ and $N$ but we really need it in the new variables, taking into account besides that $g, \nu$ are undefined for collision orbits. As an intermediate step we obtain the backward change associated to the normal form that puts the untransformed coordinates in terms of the transformed ones. The process is done in terms of the canonical set of variables introduced in [49], concretely, the angles $\ell, \theta_{a}, \phi_{a}$ and their respective conjugate actions $L, I_{2}, N$. The angle $\theta_{a}$ refers to the latitude of the aphelion while $I_{2}$ is a function that depends on the Delaunay elements $G, N$ and $g$. Note that $\left(\theta_{a}, \phi_{a}\right)$ represent the spherical polar coordinates of a unit vector pointing from the origin to the aphelion. In our setting we have replaced $\ell / L$ by $E / P_{E}$, keeping the whole set symplectic and valid near rectilinear motions. Assuming that in these variables the collision set is given by the equalities $E=0, I_{2}=N=0$, we end up with the following expression:

$$
\begin{aligned}
& \tilde{\theta}_{a}=\theta_{a}+O\left(\varepsilon^{5}\right), \quad \tilde{\phi}_{a}=\phi_{a}+O\left(\varepsilon^{5}\right), \quad \tilde{P}_{E}=P_{E}, \\
& \tilde{E}=-\frac{15}{8} \varepsilon^{3} \bar{\mu} L^{2} P_{E} \sin \left(2 \phi_{a}\right) \sin ^{2}\left(\theta_{a}\right)+O\left(\varepsilon^{5}\right), \\
& \tilde{I}_{2}=\frac{15}{16} \varepsilon^{3} \bar{\mu} L^{2} P_{E}^{2} \sin \left(2 \phi_{a}\right) \sin \left(2 \theta_{a}\right)+O\left(\varepsilon^{5}\right), \\
& \tilde{N}=\frac{15}{8} \varepsilon^{3} \bar{\mu} L^{2} P_{E}^{2} \cos \left(2 \phi_{a}\right) \sin ^{2}\left(\theta_{a}\right)+O\left(\varepsilon^{5}\right),
\end{aligned}
$$

where the tilde's variables stand for the transformed coordinates. The above formulae give the collision set in the normal form coordinates that are parameterized in terms of the untransformed ones. From now one we drop the tildes.

In the next step we replace $\theta_{a}, I_{2}$ by $\varphi, J$. The procedure is lengthy and involves carrying out the calculations in powers of $\varepsilon$ up to $\varepsilon^{3}$. We stress that $P_{E}$ is untouched as we have not made use of the generating function related to the average over $E$. After many simplifications the collision set $\mathcal{C}$ ol in the normal form variables reads as

$$
\begin{gathered}
E=-\frac{15}{8} \varepsilon^{3} \bar{\mu} L^{2} P_{E} \sin \left(2 \phi_{a}\right)+O\left(\varepsilon^{5}\right), \quad \varphi=\frac{\pi}{2}+O\left(\varepsilon^{3}\right), \\
N=\frac{15}{8} \varepsilon^{3} \bar{\mu} L^{2} P_{E}^{2} \cos \left(2 \phi_{a}\right)+O\left(\varepsilon^{4}\right) .
\end{gathered}
$$

The intersection of the 3-torus and the collision set results in

$$
\begin{gathered}
E=-\frac{15}{8} \varepsilon^{3} \bar{\mu} c_{1} L^{2} \sin \left(2 \phi_{a}\right)+O\left(\varepsilon^{5}\right), \quad \varphi=\frac{\pi}{2}+O\left(\varepsilon^{3}\right), \\
P_{E}=c_{1}+O\left(\varepsilon^{8}\right), \quad N=\frac{15}{8} \varepsilon^{3} \bar{\mu} c_{1}^{2} L^{2} \cos \left(2 \phi_{a}\right)+O\left(\varepsilon^{4}\right), \quad J=c_{3}+O\left(\varepsilon^{8}\right),
\end{gathered}
$$

together with the constraint

$$
c_{2}=\varepsilon^{3} c_{2}^{*}=\frac{15}{8} \varepsilon^{3} \bar{\mu} c_{1}^{2} L^{2} \cos \left(2 \phi_{a}\right)+O\left(\varepsilon^{4}\right)
$$

with $c_{2}^{*}>0$. Then it is deduced that $\phi_{a}$ can be fixed after putting it in terms of $c_{2}^{*}, \bar{\mu}, c_{1}$ and $L$, leading generically to four possible values, namely, $\phi_{a}^{*, i}, i=1, \ldots, 4$. Thus, the intersection of the single 3 -torus with $\mathcal{C}$ ol is given by two points, so it is of codimension 3 in the fixed torus.

Next we have to obtain the normal vector spaces to the 3 -torus and to the set $\mathcal{C}$ ol, checking that the two vectors are never parallel and thus the intersection of the manifolds is transversal. Firstly 
we sort the coordinates as $E, \phi_{a}, \varphi, P_{E}, N, J$. For the set $\mathcal{C} o l$ we get

$$
J_{\mathcal{C} o l}=\left(\begin{array}{cccccc}
1 & \varepsilon^{3} k_{1} & 0 & \varepsilon^{3} k_{2} & 0 & 0 \\
0 & 0 & 1 & 0 & 0 & 0 \\
0 & 2 \varepsilon^{3} c_{1}^{2} k_{2} & 0 & -\varepsilon^{3} k_{1} & 1 & 0
\end{array}\right)+\mathcal{O}\left(\varepsilon^{3}\right),
$$

with $k_{1}=\frac{15}{4} c_{1} L^{2} \bar{\mu} \cos \left(2 \phi_{a}^{*, i}\right), k_{2}=\frac{15}{8} L^{2} \bar{\mu} \sin \left(2 \phi_{a}^{*, i}\right)$. The corresponding normal vector at the intersection points is

$$
\mathcal{N}_{\mathcal{C o l}}=\left(s_{1}, \varepsilon^{3}\left(s_{1} k_{1}+2 s_{3} c_{1}^{2} k_{2}\right), s_{2}, \varepsilon^{3}\left(s_{1} k_{2}-s_{3} k_{1}\right), s_{3}, 0\right)+\mathcal{O}\left(\varepsilon^{3}\right),
$$

with $s_{i} \in \mathbb{R}$. For the 3 -torus the corresponding Jacobian at the intersection becomes

$$
J_{T}=\left(\begin{array}{cccccc}
0 & 0 & 0 & 1 & 0 & 0 \\
0 & 0 & 0 & 0 & 1 & 0 \\
0 & 0 & 0 & 0 & 0 & 1
\end{array}\right)+\mathcal{O}\left(\varepsilon^{3}\right)
$$

The normal vector to the 3 -torus is

$$
\mathcal{N}_{T}=\left(0,0,0, t_{1}, t_{2}, t_{3}\right)+\mathcal{O}\left(\varepsilon^{3}\right),
$$

where $t_{j}$ are real parameters. When $k_{2} \neq 0, \mathcal{N}_{T}$ and $\mathcal{N}_{\mathcal{C} o l}$ are parallel only when $s_{i}=t_{i}=0$ and the transversality condition holds. If $k_{2}=0$ then $\phi_{a}^{*, 1}=0, \phi_{a}^{*, 2}=\pi / 2, \phi_{a}^{*, 3}=\pi$ and $\phi_{a}^{*, 4}=3 \pi / 2$. However we can avoid getting $k_{2}=0$ by choosing $c_{2}^{*}$ in such a way that (6.18) does not contain the roots $0, \pi / 2, \pi, 3 \pi / 2$.

Concluding, the vectors $\mathcal{N}_{\mathcal{C} o l}$ and $\mathcal{N}_{T}$ intersect transversely, Lemma 7.4 of [52] can be applied and most of the trajectories on those tori intersecting $\mathcal{C}$ ol transversely do not intersect $\mathcal{C}$ ol. A single quasi-periodic trajectory of rectilinear equatorial character gives rise to a quasi-periodic solution of non-collision type of the system defined by Hamiltonian (6.2) that passes closely to $\left\{q_{1}=q_{2}=q_{3}=0\right\}$. These motions form a set of positive measure.

Remark 7. The transversality check performed for the two types of invariant tori is equivalent to prove that the rank of the Jacobian matrix formed by $J_{\mathcal{C} o l}$ and $J_{T}$ is maximal, which in this case is six.

Remark 8. The invariant tori we get in the non-regularised phase space are indeed invariant "punctured" tori as they have punctures that correspond to the inner collisions, where the infinitesimal particle gets arbitrarily close to its primary an infinite number of times.

\section{ACKNOWLEDGMENTS}

We appreciate the comments and suggestions of the referees that have helped to improve the manuscript.

The authors have received partial support from Projects MTM 2014-59433-C2-1-P of the Ministry of Economy and Competitiveness of Spain, from MTM 2017-88137-C2-1-P of the Ministry of Economy, Industry and Competitiveness of Spain and from the Charles Phelps Taft Foundation.

\section{REFERENCES}

1. Bacry, H., Ruegg Cern, H., and Souriau, J.-M., Dynamical Groups and Spherical Potentials in Classical Mechanics, Comm. Math. Phys., 1966, vol. 3, pp. 323-333.

2. Belbruno, E. A., A New Family of Periodic Orbits for the Restricted Problem, Celestial Mech., 1981, vol. 25, pp. 195-217.

3. Benevieri, P., Gavioli, and A., Villarini, M., Existence of Periodic Orbits for Vector Fields via Fuller Index and the Averaging Method, Electron. J. Differential Equations, 2004, vol. 2004, no. 128, pp. 1-14.

4. Besse, A. L., Manifolds all of whose geodesics are closed, Berlin Heidelberg: Springer-Verlag, 1978. 
5. Brouwer, D. and Clemence, G. M., Methods of celestial mechanics, New York and London: Academic Press, 1961.

6. Chenciner, A. and Llibre, J., A Note on the Existence of Invariant Punctured Tori in the Planar Circular Restricted Three-Body Problem, Ergodic Theory Dynam. Systems, 1988, vol. 8*, pp. 63-72.

7. Cordani, B., The Kepler problem: Group theoretical aspects, regularization and quantization, with application to the study of perturbations, Springer Basel AG, 2003.

8. Cushman, R., Reduction, Brouwer's Hamiltonian, and the Critical Inclination, Celestial Mech., 1983, no. 31, pp. 401-429. Errata, Celestial Mech., 1984, vol. 33, p. 297.

9. Cushman, R., Normal Forms for Hamiltonian Vector Fields with Periodic Flow, in Differential Geometric Methods in Mathematical Physics, S. Sternberg (Ed.), Dordrecht: D. Reidel, 1984, pp. 125-144.

10. Cushman, R., A Survey of Normalization Techniques Applied to Perturbed Keplerian Systems, in Dynamics Reported, New Series: vol. 1, C.K.R. T. Jones, U. Kirchgraber and H. O. Walther (Eds.), Berlin, Heidelberg: Springer-Verlag, 1992, pp. 54-112.

11. Cushman, R. H. and Sadovskií, D. A., Monodromy in the Hydrogen Atom in Crossed Fields, Phys. D, 2000, vol. 142, pp. 166-196.

12. Cushman, R. and Sanders, J.A., The Constrained Normal Form Algorithm, Celestial Mech., 1989, vol. 45, pp. 181-187.

13. Deprit, A., Canonical Transformations Depending on a Small Parameter, Celestial Mech., 1969, vol.1, pp. $12-30$.

14. Deprit, A., The Elimination of the Parallax in Satellite Theory, Celestial Mech., 1981, vol. 24, pp. 111153.

15. Dubrovin, B. A., Fomenko, A.T., and Novikov, S. P., Modern geometry - methods and applications. Part II. The geometry and topology of manifolds, New York: Springer-Verlag, 1985.

16. Féjoz, J., Averaging the Planar Three-Body Problem in the Neighborhood of Double Inner Collisions, J. Differential Equations, 2001, vol. 175, pp. 175-187.

17. Ferrer, S. and Lara, M., Families of Canonical Transformations by Hamilton-Jacobi-Poincaré Equation. Application to Rotational and Orbital Motion, J. Geom. Mech., 2010, vol. 2, pp. 223-241.

18. Fock, V., Zur Theorie des Wasserstoffatoms, Zeitschrift für Physik, 1935, vol. 98, pp. 145-154.

19. Goursat, M.E., Les transformations isogonales en Mécanique, Comptes rendus hebdomadaires des séances de l'Académie des sciences, 1889, vol. 53, pp. 446-448.

20. Györgyi, G., Kepler's Equation, Fock Variables, Bacry's Generators and Dirac Brackets, Il Nuovo Cimento A, 1968, vol. 108, pp.717-736.

21. Han, Y., Li, Y., and Yi, Y., Invariant Tori in Hamiltonian Systems with High Order Proper Degeneracy, Ann. Henri Poincaré, 2010, vol. 10, pp. 1419-1436.

22. Han, M. Y. and Stehle, P., $S U_{2}$ as a Classical Invariance Group, Il Nuovo Cimento A, 1967, vol.48, pp. $180-187$.

23. Heckman, G. and de Laat, T., On the Regularization of the Kepler problem, J. Symplectic Geom., 2012, vol. 10, pp. 463-473.

24. Henrard, J., Virtual Singularities in the Artificial Satellite Theory, Celestial Mech., 1974, vol. 10, pp. 437449.

25. Howison, R. C. and Meyer, K. R., Doubly-Symmetric Periodic Solutions of the Spatial Restricted ThreeBody Problem, J. Differential Equations, 2000, vol. 163, pp. 174-197.

26. Kustaanheimo, P. and Stiefel, E., Perturbation Theory of Kepler Motion Based on Spinor Regularization, J. Reine Angew. Math., 1964, vol. 218, pp. 204-219.

27. Levi-Civita, T., Sur la Régularisation du Problème des Trois Corps, Acta Math., 1920, vol. 42, pp. 99-144.

28. Ligon, T. and Schaaf, M., On the Global Symmetry of the Classical Kepler Problem, Rep. Math. Phys., 1976, vol. 9, pp. 281-300.

29. Marchal, C., Collisions of Stars by Oscillating Orbits of the Second Kind, Acta Astronaut., 1978, vol. 5, pp. $745-764$.

30. Marsden, J. and Weinstein, A., Reduction of Symplectic Manifolds with Symmetry, Rep. Math. Phys., 1974, vol. 5, pp. 121-130.

31. Meyer, K. R., Symmetries and Integrals in Mechanics, in Dynamical Systems, M. M. Peixoto (Ed.), New York: Academic Press, 1973, pp. 259-272.

32. Meyer, K. R. and Offin, D., Introduction to Hamiltonian dynamical systems and the N-Body Problem, 3rd ed., Springer International Publishing AG, 2017.

33. Meyer, K. R., Palacián, J. F., and Yanguas, P., Invariant Tori in the Lunar Problem, Publ. Mat., 2014, vol. Extra, pp. 353-394.

34. Milnor, J., On the Geometry of the Kepler Problem, Amer. Math. Monthly, 1983, vol. 90, pp. 353-365.

35. Milnor, J. and Stasheff, J. D., Characteristic classes, Princeton: Princeton University Press, 1974.

36. Moser, J., Regularization of Kepler's Problem and the Averaging Method on a Manifold, Comm. Pure Appl. Math., 1970, vol. 23, pp. 609-636.

37. Moser, J. and Zehnder, E. J. Notes on dynamical systems, Courant Lecture Notes in Mathematics, New York: New York University, 2005. 
38. Noether, E., Invariante Variationsprobleme, Nachr. V. D. Gesellsch. D. Wiss. Zu Göttingen, Math.Phys. Klasse, 1918, vol. 1918, pp. 235-257.

39. Osácar, C. and Palacián, J., Decomposition of Functions for Elliptic Orbits, Celestial Mech. Dynam. Astronom., 1994, vol. 60, pp. 207-223.

40. Palacián, J. F., Normal Forms for Perturbed Keplerian Systems, J. Differential Equations, 2002, vol. 180, pp. 471-519

41. Palacián, J. F., Sayas, F., and Yanguas, P., Regular and Singular Reductions in the Spatial Three-Body Problem, Qual. Theory Dyn. Syst., 2013, vol. 12, pp. 143-182.

42. Palacián, J. F., Sayas, F., and Yanguas, P., Flow Reconstruction and Invariant Tori in the Spatial ThreeBody Problem, J. Differential Equations, 2015, vol. 258, pp. 2114-2159.

43. Palacián, J.F., Sayas, F., and Yanguas, P., Invariant Tori of the Spatial Three-Body Problem Related to Inner Rectilinear Motions, submitted, 2018.

44. Pauli Jr., W., Über das Wasserstoffspektrum vom Standpunkt der neuen Quantenmechanik, Zeitschrift für Physik, 1926, vol. 36, pp. 336-363.

45. Reeb, G., Sur Certaines Propriétés Topologiques des Trajectoires des Systèmes Dynamiques, Acad. Roy. Sci. Lett. et Beaux-Arts de Belgique. Cl. des Sci. Mémoires in 8 $27,1952$.

46. Sayas, F., Averaging, reduction and reconstruction in the spatial three-body problem, $\mathrm{PhD}$ Thesis, Universidad Pública de Navarra, 2015.

47. Scheifele, G. and Graf, O., Analytical Satellite Theories Based on a New Set of Canonical Elements, AIA A Paper, N. 74-838, AIAA Mechanics and Control of Flight Conference, August 1974, Anaheim, California, 1974.

48. Sturmfels, B., Algorithms in invariant theory, 2nd ed., SpringerWienNewYork, 2008.

49. Tremaine, S., Canonical Elements for Collision Orbits, Celestial Mech. Dynam. Astronom., 2001, vol. 79, pp. 231-233.

50. van der Meer, J.-C. and Cushman, R., Constrained Normalization of Hamiltonian Systems and Perturbed Keplerian Motion, Z. Angew. Math. Phys., 1986, vol. 37, pp. 402-424.

51. Yanguas, P., Palacián, J. F., Meyer, K. R., and Dumas, H. S., Periodic Solutions in Hamiltonian Systems, Averaging, and the Lunar Problem, SIAM J. Appl. Dyn. Syst., 2008, vol. 7, pp. 311-340.

52. Zhao, L., Quasi-Periodic Almost-Collision Orbits in the Spatial Three-Body Problem, Commun. Pure Appl. Math., 2015, vol.68, pp. 2144-2176. 